\title{
Estructura del consejo de administración en la empresa familiar versus no familiar: evidencia empírica en España *
}

\author{
The structure of the board of directors in family \\ versus non-family firms: \\ Empirical evidence in Spain
}

\author{
Rebeca García-Ramos **. Universidad de Cantabria. \\ Myriam García Olalla. Universidad de Cantabria
}

\begin{abstract}
RESUMEN El objetivo de este trabajo es analizar la influencia de la dimensión cualitativa de la estructura de propiedad, en particular de la identidad familiar del accionista de control mayoritario, sobre la configuración óptima del Consejo de Administración, así como el efecto que dicha configuración tiene en la creación de valor de la organización. La técnica econométrica utilizada, el estimador de sistema del Método Generalizado de los Momentos, aplicada para un panel de empresas españolas familiares y no familiares cotizadas en el período 2001-2007, nos permite solventar el tradicional problema de endogeneidad presente en los estudios de gobierno corporativo. Los resultados obtenidos muestran las diferentes necesidades de supervisión y asesoramiento que tienen las empresas familiares con respecto a las de naturaleza no familiar y el consecuente efecto sobre las características estructurales y funcionales de sus consejos de administración. Entre las principales conclusiones encontramos que, a pesar de la creencia generalizada, los consejos pequeños e independientes no son siempre más eficientes.
\end{abstract}

PALABRAS CLAVE Consejo de Administración; Creación de Valor; Empresa Familiar Española; Datos de Panel; Endogeneidad.

\begin{abstract}
The aim of this paper is to analyze whether the qualitative dimension of the ownership structure, in particular the family identity of the controlling shareholder, influences the optimal configuration of the Board of Directors, as well as the effect that such configuration has on firm value creation. The econometric technique used, the system estimator of the Generalized Method of Moments, applied to a panel of Spanish family and non-family listed companies in the period 2001-2007, allows us to solve the traditional problem of endogeneity existing in studies of corporate governance. The results show how the need for advising and monitoring depends on the family nature of the controlling shareholder and, therefore, the optimal structure of the Board of Directors differs between the two classes of companies under consideration. Among the main conclusions, we found that, contrary to the widespread believe, smaller and more independent boards are not always more efficient.
\end{abstract}

KEYWORDS Board of Directors; Firm Value Creation; Spanish Family Firm; Panel Data; Endogenity.

\footnotetext{
* Los autores agradecen a los revisores anónimos su contribución a este trabajo.

* Autora para correspondencia: Rebeca García-Ramos, Departamento de Administración de Empresas, Facultad de Ciencias Económicas y Empresariales, Universidad de Cantabria, Avenida de los Castros s/n, (39005) Santander, Cantabria, España. Tel. +34 942203922, Correo-e: rebeca.garciaramos@unican.es
} 


\section{INTRODUCCIÓN}

La mayor parte de la literatura empírica existente en el ámbito del gobierno corporativo se ha centrado en empresas con estructuras de propiedad dispersas. Durante los últimos años, diversos estudios han demostrado que éstas no predominan tanto como sugieren los argumentos de Berle y Means (1932), revelando la importancia que tienen en la mayor parte de los tejidos empresariales las estructuras de propiedad concentradas en las que el accionista de control mayoritario tiene, en muchas ocasiones, naturaleza familiar. Algunos de los trabajos que han constatado la importancia de la empresa familiar son Shleifer y Vishny (1986), La Porta et al. (1999), Anderson y Reeb (2003), Anderson et al. (2003), Gómez-Mejía et al. (2003), Schulze et al. (2003) y Villalonga y Amit (2006) para el mercado norteamericano; Fernández y Nieto (2001), Faccio y Lang (2002), Galve y Salas (2003), Barontini y Caprio (2005) y Maury y Pajuste (2005) para el mercado europeo; Mok et al. (1992), Claessens et al. (2000) y Tsai et al. (2006) para el mercado asiático; y Anderson y Reeb (2003) y Mroczkowski y Tanewski (2007) para el mercado australiano.

Diversos factores motivan el desarrollo de trabajos que consideren conjuntamente la empresa familiar y el gobierno corporativo. La evidencia empírica reciente señala que el control familiar influye en la forma en que son utilizados los mecanismos internos de gobierno (Bartholomeusz y Tanewski, 2006), por lo que enmarcamos nuestro trabajo dentro del ámbito de estudio de uno de esos mecanismos, el Consejo de Administración. El papel desempeñado por éste va a tener una relevancia añadida en el caso de las empresas familiares con respecto a las no familiares, ya que, además de ocuparse de la interrelación empresa-propiedad, se va a tener que encargar de la interacción de dichas dimensiones con una variable adicional, la familia.

La creciente preocupación por encontrar modelos óptimos de gobierno ha generado un gran interés por la estructura y el funcionamiento de los consejos de administración. Así, han surgido numerosas recomendaciones acerca de su tamaño y su estructura, que se han materializado en Códigos de Buen Gobierno. Para el caso de España, el Código Conthe (2006) recomienda que, con el fin de lograr un funcionamiento eficaz y participativo, los consejos de las empresas cotizadas no tengan un tamaño inferior a cinco miembros ni superior a quince y que, al menos, una tercera parte de los consejeros sean independientes.

Desde el ámbito académico, la estructura de los consejos de administración y su impacto sobre la creación de valor de la empresa han sido abordados en numerosos estudios. La mayor parte de los mismos se han basado en una única función del consejo, la supervisión de la actuación directiva, señalando la necesidad de contar con consejos relativamente pequeños, activos y externos, incluso en las empresas con estructura de propiedad familiar (Corbetta y Salvato, 2004). La incorporación en el análisis de su función asesora arroja evidencias que sólo apoyan parcialmente estas recomendaciones, demostrando la superioridad de los consejos grandes y ejecutivos en determinados contextos de análisis en los que la función de asesoramiento puede ser más relevante que la de supervisión.

La estructura óptima del consejo está subordinada a las necesidades específicas de asesoramiento y supervisión que tiene la empresa, así como a los costes y beneficios asociados 
a esas funciones (Linck et al., 2008). Éstos no son homogéneos para todas las organizaciones, sino que dependen de determinadas características de las mismas, entre ellas sus restantes mecanismos de gobierno. Partiendo de este enfoque, nuestro principal objetivo es analizar si para una muestra de empresas españolas cotizadas en el período 2001-2007 existen diferencias significativas en el desempeño de las competencias atribuidas al consejo en función de la naturaleza familiar o no de la compañía y si aquéllas se traducen en la creación de valor de la organización.

Nuestro trabajo contribuye a la literatura existente en varios aspectos. En primer lugar, profundizamos en el conocimiento de la empresa familiar y en sus características distintivas con respecto a la no familiar, especialmente en sus prácticas de gobierno. A diferencia de la mayor parte de estudios sobre las organizaciones familiares de países europeos, que han utilizado criterios de propiedad independientes de las estructuras de gobierno, realizamos un análisis integrado en el que tenemos en cuenta ambas dimensiones. Además, utilizamos una metodología relativamente nueva y que ha sido empleada en escasos trabajos previos para la definición de la empresa familiar, la metodología de las cadenas de control.

Por otro lado, mediante la consideración conjunta de las funciones de supervisión y asesoramiento pretendemos conseguir una mejor comprensión del comportamiento de los consejos, para, en última instancia, determinar si su estructura óptima depende o no de la identidad familiar del accionista de control. Los resultados que se desprenden del análisis llevado a cabo pueden resultar de especial relevancia para las autoridades competentes en materia de elaboración de recomendaciones de buen gobierno, así como para los propietarios de empresas de naturaleza familiar.

Finalmente, con respecto a la técnica econométrica utilizada, nuestra elección viene motivada por la importancia de solventar varios problemas asociados a los estudios de gobierno corporativo, como la heterogeneidad inobservable y la potencial endogeneidad y heterocedasticidad de las variables exógenas consideradas, habitualmente omitidos en los trabajos empíricos existentes. Así mismo, demostramos la robustez y superior eficiencia de nuestras estimaciones, obtenidas con el estimador de sistema MGM en dos etapas para panel dinámico, con los reportados por otras metodologías alternativas tradicionalmente utilizadas en la literatura, como el estimador de mínimos cuadrados ordinarios y el estimador within de un modelo de efectos fijos.

A continuación, el trabajo se estructura de la siguiente manera: en el apartado segundo se revisa la literatura previa y se plantean las hipótesis; en el tercero se describe la muestra, así como la metodología y las variables empleadas; en el cuarto se presentan los principales resultados obtenidos; y se finaliza con las conclusiones y la bibliografía.

\section{REVISIÓN DE LA LITERATURA E HIPÓTESIS DE PARTIDA}

\subsection{LA ESTRUCTURA DE PROPIEDAD FAMILIAR}

La Teoría de la Agencia (Jensen y Meckling, 1976) asume que los problemas de riesgo moral $\mathrm{y}$, por tanto, los costes de agencia son menores en las empresas con estructuras de propiedad 
concentradas, dados los incentivos y mayor facilidad con la que los grandes accionistas pueden supervisar al equipo directivo. Si en éstas la dirección está en manos de los propietarios, como ocurre generalmente en las empresas familiares, se tenderá a eliminar el problema de agencia que deriva de la separación de la propiedad y el control. Los aspectos cualitativos ligados al nivel de propiedad, además de tener una especial trascendencia a la hora de determinar los conflictos de agencia a los que se va a enfrentar una determinada organización, influyen también en los objetivos que persigue. Los propietarios familiares se preocupan por la continuidad temporal de la empresa, a la que contemplan como un legado que transmitir de generación en generación, lo que les lleva a centrarse en horizontes temporales más largos que otro tipo de accionistas mayoritarios. Con el fin de mantener su negocio durante generaciones, pueden crear efectos altruistas (Dyer, 2003) que alineen los intereses de todos los participantes en el proceso de producción de valor, ya sean suministradores de capital y/o deuda, trabajo o de otros bienes y servicios ${ }^{(1)}$. De acuerdo con este argumento, la presencia de propietarios familiares y su horizonte de inversión mayor que los de las empresas con propiedad dispersa conducirán a reducir la discrecionalidad directiva, desembocando en un mayor valor de las compañías (Anderson y Reeb, 2003).

Sin embargo, las estructuras de propiedad concentradas y la presencia de grupos familiares pueden desencadenar otros problemas de gobierno. Cuando existen grandes accionistas, es más probable que surjan conflictos de intereses entre aquéllos y los minoritarios. En las empresas familiares, dadas sus mayores asimetrías informativas, la probabilidad de expropiación de los recursos corporativos es mayor, incluyendo la probabilidad de atrincheramiento de un equipo directivo familiar no cualificado (McVey et al., 2005; Sacristán y Gómez, 2007). Además, debido a su mayor preocupación por conservar el control de la empresa, es frecuente la participación de los miembros de la unidad familiar en la alta dirección, lo que puede conducir a una subordinación de los objetivos empresariales a otros familiares, como mantener el control de la compañía, la independencia financiera o la armonía familiar. Estas distorsiones llevan a algunos autores a proponer la eliminación de las influencias familiares en el seno de la empresa mediante su profesionalización (Levinson, 1971). En cambio, otra corriente de la literatura sostiene que, partiendo de que en estas empresas el conocimiento específico sobre el negocio suele estar concentrado en unos pocos agentes, los propietarios familiares, resulta más eficiente asignarles la toma de decisiones de dirección y control (Fama y Jensen, 1983).

Por todo lo expuesto, la naturaleza familiar de los accionistas de control va a determinar las necesidades particulares de gobierno de este tipo de empresas, influyendo en la forma en la que utilizan los mecanismos internos de gobierno. Su consejo de administración se convierte en un órgano que tendrá características estructurales y funcionales distintivas con respecto a la empresa no familiar.

\subsection{El CONSEJo DE ADMINISTRACión}

A partir de algunos trabajos previos (Raheja, 2005; Adams y Ferreira, 2007; Acero y Alcalde, 2008; Coles et al., 2008; Andrés y Vallelado, 2008), clasificamos las responsabilidades

(1) En la terminología anglosajona estos participantes son conocidos con el nombre de stakeholders. 
del Consejo de Administración en dos funciones principales ${ }^{(2)}$, la de supervisión y la de asesoramiento. La estructura del consejo es un factor clave para el estudio de las funciones que desempeña (Huse 2005; Voordeckers et al., 2007); así, determinadas características, como su tamaño, independencia y propiedad interna, reflejan la habilidad y capacidad de los consejeros para desempeñar eficientemente una u otra función, y, por tanto, cabe esperar que dicha composición influya sobre su eficiencia. En línea con la literatura empírica previa, aproximamos dicha eficiencia a través de la creación de valor de la empresa, bajo el argumento de que cuanto más eficiente sea el Consejo de Administración en el desempeño de sus funciones, mejor gobernada estará la empresa, lo que se traducirá en un mayor valor de la compañía (Daily y Dalton, 1993).

Para contrastar si existen diferencias significativas en el desempeño de sus competencias en función de la naturaleza familiar o no de la empresa y si dichas diferencias se traducen en la creación de valor, incorporamos en el análisis la dimensión cualitativa de la estructura de propiedad como posible mecanismo de gobierno sustitutivo del Consejo de Administración. Esperamos que las necesidades de supervisión y asesoramiento, así como los beneficios y costes asociados a dichas funciones, difieran entre empresas según la identidad familiar o no del accionista de control, lo que se verá reflejado en la estructura del consejo a través de las tres dimensiones consideradas, tamaño, independencia y propiedad interna.

\subsubsection{Hipótesis acerca de la dimensión del consejo de administración}

Desde la perspectiva de la función de supervisión, los consejos de administración deben ser reducidos. A pesar de que un mayor número de consejeros aumenta la capacidad supervisora, los consejos sobredimensionados conducen a un aumento de los costes asociados al conflicto del polizón y a los problemas de coordinación, control y flexibilidad en la toma de decisiones, lo que afecta negativamente a la eficiencia de la función de supervisión y se traduce en una menor creación de valor. La literatura empírica ha corroborado la existencia de una relación negativa entre la dimensión del consejo y diferentes medidas de la creación de valor (Yermack, 1996; Eisenberg et al., 1998; Mínguez, 2005; Cheng, 2008).

La incorporación en el análisis de la función asesora permite comprender por qué siguen existiendo consejos grandes, a pesar de la evidencia contrastada acerca de su efecto negativo en términos de supervisión. Un mayor número de miembros aporta a la empresa más capital humano, de tal forma que aumenta la información y el conocimiento específico sobre el negocio a disposición del consejo, contribuyendo al eficiente desempeño de su función asesora y, por tanto, a una mayor creación de valor. Como señalan Andrés y Rodríguez (2008), esta hipótesis es razonable si mayor número de consejeros (mayor tamaño) se asocia con mayor conocimiento y asesoramiento. Estudios recientes han demostrado que bajo determinados contextos en los que la función de asesoramiento cobra más importancia que la de supervisión, los consejos de mayor tamaño son más eficaces (Adams y Ferreira, 2007; Coles et al., 2008; Andrés y Rodríguez, 2008; Harris y Raviv, 2008; Linck et al., 2008).

(2) Al Consejo de Administración se le atribuyen numerosas tareas, véase, por ejemplo, The Business Roundtable (1990 y 2002) para una descripción detallada. 
De este modo, el efecto de la dimensión del consejo sobre la creación de valor de la empresa será un trade-off entre las ventajas (supervisión y asesoramiento) y las desventajas (problemas de coordinación, control y toma de decisiones). La relación será positiva hasta alcanzar un tamaño óptimo, a partir del cual la incorporación de un miembro adicional no aportará un mayor capital humano ni una mayor capacidad de supervisión, sino que conducirá a problemas de coordinación, control y flexibilidad en la toma de decisiones, que se traducirán en una menor creación de valor. Nuestra primera hipótesis queda enunciada como sigue:

$H_{1}$ : Existe una relación no lineal entre el tamaño del consejo y la creación de valor de la empresa.

La dimensión óptima a partir de la cual la relación entre estas variables se vuelve negativa depende de la importancia de cada una de las funciones del consejo consideradas y de sus costes y beneficios. La empresa familiar tiene una menor necesidad de supervisión, ya que, como hemos comentado, es frecuente que los miembros de la familia participen en el consejo y en la dirección, lo que conduce a una mayor alineación de intereses entre la propiedad y la dirección. De esta forma, el aumento del tamaño del consejo no aporta grandes ventajas en términos de supervisión, sino que genera problemas de control y coordinación en la toma de decisiones. Además, dada la mayor aversión al riesgo que presentan ante su mayor necesidad de conservar el control, son reacias a la entrada de agentes externos en la organización, por lo que la probabilidad de que los consejeros adicionales incorporados sean miembros de la unidad familiar es alta. Se suman así a los problemas anteriormente mencionados los potenciales conflictos de poder que puedan surgir entre los distintos miembros de la familia participantes en la empresa (Santana y Cabrera, 2001). En este sentido, los posibles beneficios derivados de un mayor asesoramiento se verán pronto compensados por los problemas que hemos expuesto, por lo que nuestra segunda hipótesis es la siguiente:

$H_{2}$ : En la empresa familiar, los costes asociados a un consejo de mayor tamaño superan antes que en la no familiar las ventajas en términos de supervisión y asesoramiento.

\subsubsection{Hipótesis acerca de la independencia del consejo de administración}

La literatura señala que para garantizar la eficacia de las funciones de supervisión y asesoramiento del consejo no es suficiente con incorporar más miembros, sino que es necesario que éstos estén dotados del conocimiento, los incentivos y las habilidades requeridas para su desarrollo. En la década de los ochenta, la inclusión de consejeros independientes no ejecutivos comenzó a recibir una atención creciente, fundamentada en su superior capacidad de supervisión de las decisiones y actividades del equipo directivo (Fama y Jensen, 1983) gracias al menor conflicto de intereses al que se enfrentan. Sin embargo, la evidencia empírica no ofrece resultados concluyentes acerca del efecto de la incorporación de consejeros externos sobre la creación de valor de la empresa, habiéndose encontrado entre ambas variables una relación positiva (Baysinger y Butler, 1985; Pearce y Zahra, 1992; Rosenstein y Wyatt, 1997; Andrés y Vallelado, 2008), negativa (Yermack, 1996, Hermalin y 
Weisbach, 1998; Linck et al. 2008) e incluso no significativa (Hermalin y Weisbach, 1991; Klein, 1998; Villalonga y Amit, 2006).

Tal y como argumenta Raheja (2005), los consejeros internos son una importante fuente de conocimiento específico sobre la empresa, aunque pueden tener objetivos distorsionados debido a los beneficios privados y a su falta de independencia del CEO ${ }^{(3)}$. Dada su estrecha vinculación con éste, que es su supervisor directo, no son candidatos óptimos para desarrollar la función de control, ya que podrían no emitir evaluaciones críticas de sus políticas y estrategias. Los que más eficientemente pueden desarrollar esta función son aquéllos que no mantienen ningún vínculo personal ni profesional con la empresa, los externos. De este modo, si la función de supervisión prevalece sobre la de asesoramiento, esperamos una relación positiva entre independencia del consejo y creación de valor de la empresa.

Además de controlar el oportunismo gerencial, los consejeros externos son relevantes para la función asesora del consejo en cuanto que aportan un amplio conocimiento que puede ser utilizado por el equipo directivo en la formulación e implementación de sus estrategias. Sin embargo, un consejo formado íntegramente por externos no podría desempeñar eficientemente esta labor, dada su falta de conocimiento sobre aspectos fundamentales de la empresa y de su entorno (Ford, 1992). En cambio, los consejeros internos ejecutivos facilitan la transferencia de información entre el consejo y el equipo directivo. El CEO, que tiene un acceso rápido a toda la información relativa a la marcha del negocio, será reacio a compartirla con personas ajenas a él, como los externos, mientras que mostrará una mayor colaboración con los internos ejecutivos, que trabajan en su equipo en el día a día. Sin la participación de los ejecutivos, el CEO contaría con una ventaja considerable de asimetría informativa y el consejo no tendría acceso a toda la información. Atendiendo a la función asesora, los consejeros internos son una valiosa fuente de conocimiento sobre el funcionamiento de la compañía, lo que les valida como candidatos que pueden desarrollarla eficientemente. Desde esta perspectiva, esperamos una relación negativa entre independencia del consejo y creación de valor de la empresa.

Por todo lo expuesto, parece que existen conclusiones contrapuestas acerca de la participación de internos en el consejo, un argumento en contra basado en su falta de independencia del CEO y su consecuente dificultad para supervisar y controlar su actuación, y otro a favor basado en la necesidad de la empresa de contar con conocimiento de alta calidad y experiencia en el negocio. Un consejo formado íntegramente por consejeros externos podría no cumplir de forma adecuada la función de asesoramiento, al mismo tiempo que si estuviera dominado por internos su capacidad de supervisión y control podría ser inferior. Dado que la maximización de la creación de valor requiere que todas las funciones del consejo se desarrollen de forma efectiva, una combinación adecuada de externos e internos puede ser la mejor composición (Baysinger y Butler, 1985; Andrés y Vallelado, 2008), argumento que da lugar a nuestra tercera hipótesis:

$H_{3}$ : Existe una relación no lineal entre la independencia del consejo y la creación de valor de la empresa.

(3) Máximo ejecutivo de la compañía, por sus siglas en inglés, Chief Executive Officer. 
Si bien la proporción óptima de consejeros internos y externos dependerá de las características de cada empresa, existen pruebas concluyentes de que la apuesta por la total independencia no garantiza altos niveles de creación de valor, sobre todo si dicha independencia se logra a expensas de otras tareas del consejo. Los consejeros externos de las empresas familiares serán más propensos a estar influenciados por vínculos personales con los miembros familiares y por la expectativa de servir como apoyo a la dirección en la toma de decisiones, lo que puede afectar a su independencia y en consecuencia a su capacidad de supervisión y asesoramiento. Maug (1996) demuestra que para aquellas empresas que tienen importantes asimetrías de información, como es el caso de las familiares, no es óptimo incrementar la supervisión a través de la incorporación de externos, ya que resultará costosa la transferencia de conocimientos específicos sobre la empresa a personas ajenas a ella. En cambio, el CEO y el equipo directivo se caracterizan por un alto grado de compromiso hacia la empresa y por la coincidencia de sus valores con los de ésta. En este contexto y aceptando como función principal del consejo la de asesoramiento, podría ser recomendable para estas organizaciones la adopción de consejos compuestos principalmente por internos o por otro tipo de consejeros que mantengan con la organización empresarial vínculos sociales y familiares. En esta misma línea, algunos trabajos empíricos concluyen una menor necesidad de independencia cuando se produce una alineación de intereses entre los consejeros internos y el CEO respecto al resto de accionistas (Boone et al., 2007; Linck et al., 2008), o muestran cómo un mayor peso de los internos es valorado positivamente por el mercado (Andrés y Rodríguez, 2008). Nuestra cuarta hipótesis queda enunciada como sigue:

$H_{4}$ : Las ventajas de la independencia del consejo en términos de supervisión y asesoramiento son menores en las empresas familiares.

\subsubsection{Hipótesis acerca de la propiedad de los internos}

La función asesora del Consejo de Administración puede ser más visible en empresas con menores necesidades de control gracias a la supervisión ejercida por otras fuerzas alternativas, como la existencia de grandes accionistas. La forma en la que ejerce su poder un accionista de control es, a menudo, a través de su participación en el consejo. Dicha participación es mayor cuando el accionista de control tiene naturaleza familiar, encontrándose una alta convergencia entre propiedad de la unidad familiar y propiedad de los internos. La propiedad interna tiene un efecto positivo sobre la creación de valor, ya que reduce la necesidad de supervisión del equipo directivo y permite una mayor dedicación a la función de asesoramiento.

Los trabajos empíricos existentes no siempre han encontrado evidencia de su influencia significativa sobre el valor de la empresa (Demsetz, 1983; Demsetz y Villalonga, 2001). Los consejeros pueden utilizar la propiedad interna para evitar su cese en caso de comportamientos ineficientes (hipótesis de atrincheramiento), derivándose una relación negativa con la creación de valor. La participación en el capital de la sociedad por parte de aquéllos puede conllevar que otros mecanismos de control, como puede ser el mercado o el resto de accionistas, pierdan eficiencia.

Teniendo en cuenta estos planteamientos, puede existir una relación no monotónica entre ambas variables que equilibre los dos efectos anteriores, convergencia y atrincheramiento. Esta 
forma funcional, que ha sido hallada en varios trabajos (Demsetz y Lehn, 1985; Gedajlovic y Shapiro, 1998; Thomsen y Pedersen, 2000; Pindado et al., 2008), implica una relación positiva para los niveles bajos de propiedad como resultado de la hipótesis de supervisión, y negativa a partir de un determinado nivel como consecuencia de la hipótesis de expropiación.

Aparte de la evidencia empírica, los vínculos familiares pueden explicar por sí mismos esta no linealidad en términos de beneficio y coste potencial de la propiedad familiar. Cabe esperar que ambos efectos sean más acusados en este tipo de empresas. La relación positiva se verá acentuada por los efectos altruistas que se generan como consecuencia de sus horizontes de inversión mayores, mientras que la relación negativa lo hará por la mayor probabilidad de expropiación. Los inconvenientes de tener una familia como principal accionista de la empresa es más probable que surjan cuando su participación en la empresa es demasiado elevada, contribuyendo a la mejora de la creación de valor mientras la propiedad familiar aumenta y empeorándola a partir de un determinado nivel de concentración, que Anderson y Reeb (2003) sitúan en torno a un tercio.

De esta forma, en la empresa familiar esperamos la existencia de un límite al efecto positivo de la propiedad interna sobre la creación de valor, a partir del cual un incremento adicional da lugar a una posición atrincherada de la familia, disminuyendo dicha creación de valor. En las empresas no familiares, en las que la participación de los consejeros en el capital es menor, esperamos la prevalencia de la hipótesis de convergencia sobre la de atrincheramiento. Nuestras hipótesis quinta y sexta son las siguientes:

$H_{5}$ : Existe una relación positiva entre la propiedad de los consejeros y la creación de valor de la empresa.

$H_{6}$ : En las empresas familiares, existe una relación no lineal entre la propiedad de los consejeros y la creación de valor.

\section{CONTRASTE EMPÍRICO}

\subsection{COMPOSICIÓN Y CARACTERÍSTICAS DE LA MUESTRA}

La muestra utilizada para el análisis empírico se construye con empresas no financieras cotizadas en la bolsa española para el período 2001-2007. Compuesta inicialmente por 119 empresas es sometida a una serie de filtros, eliminándose aquellas para las cuales no se dispone de información sobre todas las variables necesarias para el análisis o no se dispone para un número suficiente de años de acuerdo con la técnica econométrica utili$z^{2}{ }^{(4)}$, realizan algún tipo de servicio financiero o no es posible la identificación del último propietario, según la definición que presentamos a continuación, de forma que finalmente queda constituida por 85 empresas con estructura de propiedad concentrada.

La información sobre la estructura de propiedad la obtenemos de la base Amadeus y de la información depositada por las empresas en la CNMV acerca de sus accionistas signi-

(4) Necesitamos datos disponibles para al menos cuatro años consecutivos durante el período 2001-2007 para contrastar la existencia de correlación serial de segundo orden (Arellano and Bond, 1991), prueba fundamental para garantizar la robustez de nuestras estimaciones realizadas con la metodología de sistema del Método Generalizado de los Momentos. 
ficativos; los datos sobre la dimensión y estructura del Consejo de Administración de los Informes de Gobierno Corporativo de la CNMV; la información económico-financiera de las cuentas anuales de las empresas y la información de mercado de la Bolsa de Madrid, utilizándose como fuente complementaria la base Amadeus.

Siguiendo a La Porta et al. (1999) utilizamos la metodología de las cadenas de control para la identificación del último propietario de las empresas seleccionadas. Ésta permite comprender las relaciones de propiedad existentes en la mayoría de países, dado que las empresas suelen estar controladas a través de un encadenamiento jerárquico de sociedades. Mediante su empleo evitamos la atribución del control de una compañía a un accionista que no se sitúa al final de las relaciones de propiedad, lo que podría ser especialmente grave en entornos de propiedad concentrada, donde los aspectos cualitativos asociados a la propiedad pueden ser, como hemos justificado en la revisión de la literatura, un factor determinante para entender el comportamiento y los objetivos corporativos.

Analizando la totalidad de la cadena de propiedad, consideramos que una empresa tiene último propietario cuando el principal accionista a través del último eslabón de la cadena posee directa o indirectamente un porcentaje de participación en la propiedad igual o superior a un nivel de control establecido. Aunque en la literatura se han empleado habitualmente niveles del 10\% y del 20\%, en este estudio establecemos un 25\% (Ampenberger et al., 2009), que justificamos con dos argumentos. En primer lugar para tratar de ajustarlo a las estructuras de propiedad más concentradas existentes en países como España, y en segundo término, para adoptar la definición oficial de Empresa Familiar, aprobada el 7 de Abril de 2008 por las dos principales instituciones internacionales que representan a la Empresa Familiar, el Grupo Europeo de Empresas Familiares (GEEF) en Bruselas, y el Board de la Family Business Network en Milán. Así, identificamos aquellas empresas que tienen último propietario y éste es una familia.

La mayor parte de estudios previos consideran la estructura de propiedad de forma independiente a la estructura de gobierno. Nosotros exigimos que la propiedad familiar esté representada en el Consejo de Administración para considerar a la empresa como familiar, ya que su mera participación en la propiedad no tiene por qué conllevar su implicación real en el funcionamiento de la organización, sino que se requiere la participación activa en los órganos de decisión y control. Para la muestra analizada, un 85\% de las empresas que cuentan con al menos un $25 \%$ de la propiedad en manos de grupos familiares tienen presencia en el Consejo de Administración.

Con el fin de obtener una muestra de empresas lo más homogénea posible, para así atribuir las diferencias encontradas a la identidad familiar del accionista de control, sólo se incluyen en la muestra final aquéllas que tienen último propietario identificado a través del último eslabón de la cadena, exigiéndose, como ya hemos comentado, la propiedad de al menos un $25 \%$. Atendiendo a este criterio, la muestra analizada está compuesta por un $40 \%$ de empresas familiares y un $60 \%$ no familiares, proporción similar a la reportada por Santana y Cabrera (2001). Las diferencias encontradas con Faccio y Lang (2002) y Santana y Aguiar (2006), que reportan respectivamente una proporción de empresas familiares del $60 \%$ y del $50 \%$, se deben a la muestra seleccionada y a los criterios utilizados para la definición de último propietario. 
La distribución sectorial de la muestra final puede verse en la Tabla 1.

TABLA 1

DisTRIBUCIÓN SECTORIAL DE LA MUESTRA

\begin{tabular}{|l|c|c|c|}
\hline \multicolumn{1}{|c|}{ Clasificación Sectores, Código Primario SIC } & Muestra completa & Familiares & No Familiares \\
\hline S1 Agricultura, Silvicultura y Pesca & 1 & 0 & 1 \\
\hline S2 Minería & 2 & 0 & 2 \\
\hline S3 Construcción y Contratas & 14 & 7 & 7 \\
\hline S4 Fabricantes & 23 & 9 & 14 \\
\hline S5 Transportes, Comunicaciones y Servicios Pcos, & 16 & 4 & 12 \\
\hline S6 Mayoristas & 3 & 1 & 2 \\
\hline S7 Detallistas & 1 & 1 & 0 \\
\hline S8 Seguros y Bienes Raíces & 13 & 6 & 7 \\
\hline S9 Servicios & 12 & 6 & 6 \\
\hline Total & 85 & 34 & 51 \\
\hline
\end{tabular}

\subsection{VARIABLES Y ESTADÍSTICA DESCRIPTIVA}

\subsubsection{Variable dependiente}

Nuestro objetivo es evaluar si la estructura del Consejo de Administración tiene algún impacto sobre la creación de valor de la empresa y si el potencial efecto depende de la estructura de propiedad, en concreto de su dimensión cualitativa. Para tal fin, utilizamos como variable dependiente la $Q$ de Tobin, definida como el valor de mercado de la empresa sobre el valor de reposición de los activos. Al igual que en numerosos trabajos, es aproximada a través de la Q-ratio (market-to-book ratio) ${ }^{(5)}$, calculada como el valor de mercado de los fondos propios y de la deuda entre el valor contable de los activos.

Adicionalmente, y con el propósito de comprobar la robustez de los análisis efectuados, utilizamos como medida de la variable dependiente la ratio valor, definida como el valor de mercado de las acciones sobre su valor contable.

\subsubsection{Variables explicativas}

Nuestras variables explicativas son la dimensión del consejo, aproximada a través del número de consejeros que integran el Consejo de Administración; su independencia, medida a través de la proporción de consejeros independientes sobre el total de consejeros, y la propiedad interna, aproximada por la proporción de derechos en manos del consejo.

(5) Es frecuente en la literatura sobre gobierno corporativo la utilización de esta medida u otras similares como variable dependiente (Hermalin y Weisbach, 1991; Adams y Mehran, 2005; Caprio et al., 2007, y Andrés y Vallelado, 2008). Sus problemas potenciales son el empleo del valor contable de la deuda en lugar de su valor de mercado y la utilización del valor contable para medir el coste de reemplazamiento del capital. Sin embargo, la correlación entre esta medida de la $Q$ y una medida que utilice el valor de mercado es alta (Loderer y Martín, 1997). De acuerdo con Perfect y Wiles (1994) y Chung y Pruitt (1994) es de 0,93 y 0,97 respectivamente. 


\subsubsection{Variables de control}

Como variables de control utilizamos el tamaño de la empresa, definido como el logaritmo neperiano del valor del activo total de la empresa, y su nivel de endeudamiento, aproximado por el peso de la deuda sobre el activo total.

Además, incluimos seis dummies temporales representativas de la adscripción de cada observación a un determinado año, que toma valor 1 cuando la observación muestral corresponde al año $m$ y 0 en caso contrario.

Por último, definimos la variable familiar como una dummy que adopta el valor 1 si la empresa es clasificada como familiar y 0 en caso contrario, y la incorporamos en el modelo de forma interactiva para recoger el cambio potencial que se pueda producir en los coeficientes estimados para las variables independientes.

\subsubsection{Estadística descriptiva}

En la Tabla 2 presentamos los estadísticos descriptivos de las variables utilizadas. Como puede observarse, además de las anteriormente definidas, aparecen tres nuevas variables. A diferencia de los trabajos desarrollados en el contexto anglosajón, donde suele distinguirse únicamente entre consejeros internos y externos, las especificidades del sistema de propiedad de las empresas españolas, caracterizado por una elevada concentración accionarial, nos permiten diferenciar tres tipos de miembros en los consejos de nuestras empresas: ejecutivos, dominicales e independientes. Los primeros son aquellos que detentan un cargo directivo en la compañía y, por tanto, están implicados en la gestión de la misma. Los dominicales representan los intereses de grupos accionariales con capacidad de influir en el control de la sociedad. Y, finalmente, son consejeros independientes los que no están vinculados con el equipo de gestión ni con los grupos accionariales de control, sino que, teóricamente, son designados por su alta capacitación profesional. Los dos últimos tipos de consejeros, dominicales e independientes, frente a los consejeros ejecutivos o internos, podrían asimilarse a lo que la literatura anglosajona denomina external directors 0 non-executive directors (García y Gill, 2005). Teniendo en cuenta estos argumentos, hemos incluido en la tabla los estadísticos descriptivos para tres nuevas variables, los consejeros ejecutivos (proporción de ejecutivos sobre el total de consejeros), consejeros dominicales (proporción de dominicales sobre el total de consejeros) y consejeros externos (proporción de consejeros independientes y dominicales sobre el total de consejeros).

TABLA 2

ESTADÍSTICOS DESCRIPTIVOS MUESTRA COMPLETA

\begin{tabular}{|l|r|r|r|r|c|}
\hline \multicolumn{1}{|c|}{ Variable } & $N$ & Media & Máximo & Mínimo & Desv. típ. \\
\hline Q-Tobin & 451 & 1,53 & 16,20 & 0,10 & 1,39732 \\
\hline Ratio valor & 451 & 2,84 & 108,26 & 0,02 & 6,36460 \\
\hline Dimensión consejo & 487 & 9,60 & 22,00 & 1,00 & 4,58548 \\
\hline Independencia consejo & 424 & 0,26 & 1,00 & 0,00 & 0,21894 \\
\hline
\end{tabular}

(Continúa pág. sig.) 
TABLA 2 (cont.)

ESTADÍSTICOS DESCRIPTIVOS MUESTRA COMPLETA

\begin{tabular}{|l|c|c|c|c|c|}
\hline \multicolumn{1}{|c|}{ Variable } & $N$ & Media & Máximo & Mínimo & Desv. típ. \\
\hline Consejeros ejecutivos & 425 & 0,27 & 1,00 & 0,00 & 0,21033 \\
\hline Consejeros dominicales & 424 & 0,44 & 1,00 & 0,00 & 0,26449 \\
\hline Consejeros externos & 459 & 0,69 & 1,00 & 0,00 & 0,30999 \\
\hline Propiedad interna & 390 & 0,34 & 1,00 & 0,00 & 0,32100 \\
\hline Tamaño empresa (Ln AT) & 574 & 12,77 & 17,88 & 5,90 & 2,21266 \\
\hline Endeudamiento & 565 & 0,56 & 0,93 & 0,01 & 0,22146 \\
\hline
\end{tabular}

TABLA 3

ESTADÍSTICOS DESCRIPTIVOS POR SUBMUESTRAS [EMPRESAS FAMILIARES (EF) Y NO FAMILIARES (ENF)]

\begin{tabular}{|c|c|c|c|c|c|c|}
\hline Variable & Media EF & Mediana EF & Media ENF & Mediana ENF & Z & $p$-valuet+ \\
\hline Q-Tobin & 1,49 & 1,16 & 1,55 & 1,20 & $-0,327$ & 0,743 \\
\hline Ratio valor & 2,25 & 1,47 & 3,22 & 1,54 & $-0,431$ & 0,666 \\
\hline Dimensión consejo & 8,26 & 7,00 & 10,49 & 10,00 & $-5,759$ & $0,000^{\text {** }}$ \\
\hline Independencia consejo & 0,28 & 0,28 & 0,25 & 0,25 & $-1,609$ & 0,108 \\
\hline Consejeros ejecutivos & 0,34 & 0,33 & 0,22 & 0,17 & $-7,180$ & $0,000^{* * *}$ \\
\hline Consejeros dominicales & 0,36 & 0,33 & 0,49 & 0,50 & $-5,289$ & $0,000^{* * *}$ \\
\hline Consejeros externos & 0,62 & 0,65 & 0,74 & 0,82 & $-6,490$ & $0,000^{\text {***}}$ \\
\hline Propiedad interna & 0,47 & 0,54 & 0,25 & 0,09 & $-6,036$ & $0,000^{* * *}$ \\
\hline Tamaño empresa (Ln AT) & 12,30 & 11,96 & 13,05 & 13,10 & $-3,893$ & $0,000^{* * *}$ \\
\hline Endeudamiento & 0,53 & 0,53 & 0,57 & 0,59 & $-1,225$ & 0,221 \\
\hline
\end{tabular}

+ Basado en el test U de Mann-Withney, bajo la hipótesis nula de que no existen diferencias entre ambas submuestras. Significatividad al $1 \%{ }^{* * *}$ de la diferencia entre ambas submuestras.

El tamaño medio del consejo de las empresas analizadas es de 9,6 miembros y la proporción media de consejeros ejecutivos de un $26,69 \%$, frente al $69,04 \%$ de los consejeros externos. Diferenciando dentro de los externos entre consejeros independientes y dominicales, mientras los primeros representan un porcentaje similar a los ejecutivos, $26,49 \%$, lo segundos tienen una representación mayoritaria, con un 44,14\%. Nuestros resultados son similares a los reportados por Acero y Alcalde (2008) para una muestra de empresas españolas cotizadas en el año 2005, que encuentran un tamaño medio de 10,1 miembros, y los siguientes porcentajes medios de representación de consejeros: internos ejecutivos 22,6\% y externos $77,4 \%$ (de los cuales un $44,3 \%$ son dominicales y un $30,1 \%$ independientes).

En la Tabla 3 se presentan las medias y medianas para cada una de las dos submuestras de empresas, familiares y no familiares. La variable dependiente no difiere estadísticamente entre ambas submuestras para ninguna de las dos medidas utilizadas, la Q-ratio y la ratio valor. Sin embargo, tal y como tradicionalmente ha encontrado la literatura empírica pre- 
via, tanto el tamaño medio del Consejo de Administración como la proporción de consejeros externos son significativamente menores en las empresas familiares. Si distinguimos entre los dos tipos de consejeros externos, mientras los dominicales representan un mayor peso en la empresa no familiar, siendo la diferencia estadísticamente significativa, la media de independientes no difiere significativamente entre ambas submuestras. Por último, tanto la proporción media de consejeros internos ejecutivos como la propiedad interna son significativamente mayores en las empresas familiares. Finalmente, se confirma el menor tamaño que alcanzan las familiares, mientras que no existen diferencias significativas entre ambas submuestras en cuanto a su nivel de endeudamiento.

\subsection{Metodología y ESPECIFICACIÓN DEL MODELO}

La metodología utilizada para el contraste de las hipótesis planteadas es la de datos de panel. Su principal ventaja es que permite modelizar la heterogeneidad inobservable que existe entre distintas empresas mediante la descomposición del término de error en tres componentes ${ }^{(6)}$. Además, conseguimos alargar la muestra, lo que significa un aumento sustancial de las observaciones, de los grados de libertad del modelo y, por tanto, de la consistencia de los resultados.

Un aspecto que complica los análisis empíricos sobre los consejos de administración es que la mayor parte de las variables de interés vienen determinadas endógenamente (Hermalin y Weisbach, 2003; Boone et al., 2007; Andrés y Vallelado, 2008; Linck et al., 2008), es decir, están correlacionadas con el término de error. Gran parte de la literatura empírica existente ha omitido este hecho, lo que hace que los resultados obtenidos sean difíciles de interpretar. Así, la estructura pasada del consejo afectará a la creación de valor actual de la empresa, lo que nos lleva a estimar nuestro modelo con el procedimiento de panel dinámico, que sólo asume exogeneidad débil, es decir, dependencia de las observaciones pasadas, pero no de las futuras. En este caso, tanto el estimador de Mínimos Cuadrados Ordinarios como el de efectos fijos (Within), y por tanto también el de efectos aleatorios, estarán sesgados, por lo que recurrimos al empleo de variables instrumentales para estimar el modelo de forma consistente (Arellano y Bond, 1991). Para calcular el vector de parámetros empleamos el estimador de sistema (system estimator) del Método Generalizado de los Momentos (MGM), que permite introducir en el modelo variables invariantes en el tiempo ${ }^{(7)}$, que serían eliminadas en el modelo en diferencias inicial. Además, ofrece estimaciones mucho menos sesgadas que el estimador del modelo en diferencias en el caso de que la variable dependiente sea persistente ${ }^{(8)}$, ya que en este último caso los instrumentos parecen ser débiles ${ }^{(9)}$ (Blundell y Bond, 1998). En general se recomienda que el número de instrumentos no sobrepase el de unidades de sección cruzada ${ }^{(10)}$. Por último,

(6) $u_{i t}=\eta_{i}+\lambda_{\tau}+v_{k}$ donde $\eta_{i}$ es la parte del error específica del individuo $i$ (heterogeneidad inobservable) y que recoge aquellos efectos no observables que afectan sólo a la empresa $i ; \lambda$, representa aquellos shocks que ocurren en el momento $t$ y afectan a todos los individuos por igual, y $v_{i t}$ es una perturbación aleatoria.

(7) Algunas de las variables referentes al consejo presentan una escasa variabilidad temporal.

(8) Se dice que la variable $y_{i t}$ es persistente si el parámetro a en el modelo $y_{i t}=a y_{i t-1}+u_{i t}$ se acerca a 1.

(9) Véase Bond (2002) para una discusión sobre el tema.

(10) Roodman (2009) afirma que un número excesivo de instrumentos puede ser casi tan perjudicial como la elección de elementos no idóneos y, como regla práctica, aconseja un valor para el estadístico de Hansen entre 0,1 y 0,25, y considerar 
realizamos la estimación de los parámetros en dos etapas, ya que este método es robusto ante la presencia de heterocedasticidad ${ }^{(11)}$ y correlación sectorial sea cual sea su forma. Dado que el estimador en dos etapas subestima los errores estándar de los parámetros, hemos aplicado la corrección de la matriz de varianzas y covarianzas para muestras finitas propuesta por Windmeijer (2005).

Siguiendo esta metodología, comenzamos estimando un modelo general que explica la creación de valor de la empresa en función de las variables relativas al Consejo de Administración (1). Adicionalmente, se construye un segundo modelo en el que se incluyen los cuadrados de dichas variables (2), con el fin de contrastar la existencia de relaciones no lineales para el conjunto de la muestra. Para determinar si hay o no diferencias significativas entre las empresas familiares y las no familiares incorporamos en los modelos anteriores la variable familiar de forma interactiva (3)(4). Finalmente, atendiendo a las relaciones puestas de relieve por las regresiones anteriores, se estiman los modelos finales para cada una de las dos submuestras. El subíndice $i$ hace referencia a la empresa $(i=1, \ldots . .85)$ y el subíndice $t$ al período de tiempo $(t=2001, \ldots, 2007)$.

CREACIÓN VALOR $_{i, t}=\alpha+\beta_{1}$ DIMENSIÓN CONSEJO $_{i t}+$ $\beta_{2}$ INDEPENDENCIA CONSEJO $_{i t}+\beta_{3}$ PROPIEDAD INTERNA $_{i t}+$ $\beta_{4}$ ENDEUDAMIENTO $_{i t}+\beta_{5}$ TAMAÑO EMPRESA $A_{i t}+\eta_{i}+\lambda_{t}+v_{i t}$

CREACIÓN VALOR $_{i, t}=\alpha+\beta_{1}$ DIMENSIÓN CONSEJO $_{i t}+\beta_{2}$ DIMENSIÓN CONSEJO $_{i t}{ }_{i t}+$ $\beta_{3}$ INDEPENDENCIA CONSEJO $_{i t}+\beta_{4}$ INDEPENDENCIA CONSEJO $_{i t}+$

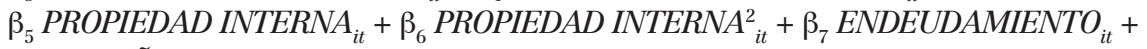
$\beta_{8}$ TAMAÑO EMPRESA $A_{i t}+\eta_{i}+\lambda_{\tau}+v_{i t}$

CREACIÓN VALOR $_{i, t}=\alpha+\beta_{1}$ DIMENSIÓN CONSEJO $_{i t}+$ $\beta_{1 F}$ DIMENSIÓN CONSEJO $_{i t}$ FAMILIAR $_{i t}+\beta_{2}$ INDEPENDENCIA CONSEJO $_{i t}+$ $\beta_{2 F}$ INDEPENDENCIA CONSEJO $_{i t}$ FAMILIAR $_{i t}+\beta_{3}$ PROPIEDAD INTERNA $_{i t}+$ $\beta_{3 F}$ PROPIEDAD INTERNA $_{i t}$ FAMILIAR $_{i t}+\beta_{4}$ ENDEUDAMIENTO $_{i t}+$ $\beta_{4 F}$ ENDEUDAMIENTO $_{i t}$ FAMILIAR $_{i t}+\beta_{5}$ TAMAÑO EMPRESA ${ }_{i t}+\beta_{5 F}$ TAMAÑO EMPRESA ${ }_{i t}$ $F A M I L I A R_{i t}+\eta_{i}+\lambda_{t}+v_{i t}$

CREACIÓN VALOR ${ }_{i, t}=\alpha+\beta_{1}$ DIMENSIÓN CONSEJO $_{i t}+\beta_{1 F}$ DIMENSIÓN CONSEJO $_{i t}$ FAMILIAR $_{i t}+\beta_{2}$ DIMENSIÓN CONSEJO ${ }_{i t}+\beta_{2 F}$ DIMENSIÓN CONSEJO $^{2}{ }_{i t}$ FAMILIAR $_{i t}$ $+\beta_{3}$ INDEPENDENCIA CONSEJO $_{i t}+\beta_{3 F}$ INDEPENDENCIA CONSEJO $_{i t}$ FAMILIAR $_{i t}+$ $\beta_{4}$ INDEPENDENCIA CONSEJO $_{i t}+\beta_{4 F}$ INDEPENDENCIA CONSEJO $_{i t}^{2}$ FAMILIAR $_{i t}+$

$\beta_{5}$ PROPIEDAD INTERNA ${ }_{i t}+\beta_{5 F}$ PROPIEDAD INTERNA $_{i t}$ FAMILIAR $_{i t}+$

$\beta_{6}$ PROPIEDAD INTERNA ${ }_{i t}^{2}+\beta_{6 F}$ PROPIEDAD INTERNA ${ }_{i t}^{2}$ FAMILIAR $_{i t}+$

$\beta_{7}$ ENDEUDAMIENTO $_{i}+\beta_{7 F}$ ENDEUDAMIENTO $_{i t}$ FAMILIAR $_{i t}+$

$\beta_{8}$ TAMAÑO EMPRESA $A_{i t}+\beta_{8 F}$ TAMAÑO EMPRESA $A_{i t}$ FAMILIAR $_{i t}+\eta_{i}+\lambda_{t}+v_{i t}$

valores excesivos del estadístico (cercanos a 1) como indicador de la existencia de problemas en el modelo, uno de los cuales podría ser la presencia de raíces unitarias en la variable dependiente.

(11) Mediante el contraste de heterocedasticidad ampliado de Wald se comprueba que nuestra muestra presenta este problema: $H_{0}: \sigma(i)^{2}=\sigma^{2}$ for all $i, \chi^{2}(85)=2,7 \mathrm{e}+06$ Prob, $>\chi^{2}=0,0000$. 


\section{RESULTADOS OBTENIDOS}

Para contrastar la validez de nuestras especificaciones hemos realizado el test de Hansen/ Sargan de sobreidentificación de los instrumentos, verificándose en todos los casos la validez de los instrumentos utilizados. Así mismo, presentamos los estadísticos $A R_{1}$ y $A R_{2}$, rechazándose la existencia de autocorrelación serial de primer y segundo orden. El test de la F muestra la significación global de las regresiones efectuadas. Para todos los modelos estimados se presentan las estimaciones MGM de sistema en dos etapas con la corrección robusta para muestras finitas propuesta por Windmeijer (2005).

\subsection{Resultados para la muestra completa}

En la Tabla 4 se presentan los resultados para la muestra completa, para la que se contrastan las hipótesis generales $H_{1}, H_{3}$ y $H_{5}$. A la vista de las estimaciones de los Modelos (1) y (2) se plantea el modelo final (1-2).

En primer lugar, el coeficiente de la variable dimensión consejo sugiere la existencia de una relación negativa y significativa entre el tamaño del consejo y la creación de valor de la empresa, por lo que en este primer nivel de análisis no podemos aceptar nuestra primera hipótesis, que establecía una relación cuadrática.

\section{TABLA 4}

ESTIMACIONES PARA LA MUESTRA COMPLETA

\begin{tabular}{|c|c|c|c|}
\hline Q-TOBIN & (1) & (2) & $(1-2)$ \\
\hline constante & $\begin{array}{l}-0,5604634 \\
(0,754)\end{array}$ & $\begin{array}{l}1,966602 \\
(0,195)\end{array}$ & $\begin{array}{l}-0,0700651 \\
(0,973)\end{array}$ \\
\hline dimensión consejo & $\begin{array}{l}-0,1370594 \\
(0,007)^{\star * *}\end{array}$ & $\begin{array}{l}-0,1755242 \\
(0,531)\end{array}$ & $\begin{array}{l}-0,0973548 \\
(0,043)^{\star *}\end{array}$ \\
\hline dimensión consejo ${ }^{2}$ & & $\begin{array}{l}0,0032209 \\
(0,710)\end{array}$ & \\
\hline independencia consejo & $\begin{array}{l}1,696932 \\
(0,103)\end{array}$ & $\begin{array}{l}3,061628 \\
(0,075)^{*}\end{array}$ & $\begin{array}{c}5,695344 \\
(0,034)^{\star *}\end{array}$ \\
\hline independencia consejo ${ }^{2}$ & & $\begin{array}{l}-4,814638 \\
(0,009)^{* * *}\end{array}$ & $\begin{array}{l}-6,821998 \\
(0,013)^{\star *}\end{array}$ \\
\hline propiedad interna & $\begin{array}{l}0,6500418 \\
(0,388)\end{array}$ & $\begin{array}{l}2,047095 \\
(0,097)^{*}\end{array}$ & $\begin{array}{c}4,062537 \\
(0,009)^{\star \star \star}\end{array}$ \\
\hline propiedad interna $^{2}$ & & $\begin{array}{l}-2,479572 \\
(0,061)^{*}\end{array}$ & $\begin{array}{l}-3,835319 \\
(0,039)^{\star *}\end{array}$ \\
\hline endeudamiento & $\begin{array}{l}-0,5063845 \\
(0,636)\end{array}$ & $\begin{array}{l}-1,517136 \\
(0,294)\end{array}$ & $\begin{array}{l}-1,264146 \\
(0,229)\end{array}$ \\
\hline tamaño empresa & $\begin{array}{c}0,3262118 \\
(0,005)^{\star * *}\end{array}$ & $\begin{array}{l}0,2202629 \\
(0,280)\end{array}$ & $\begin{array}{l}0,2310369 \\
(0,045)^{\star *}\end{array}$ \\
\hline año 2007 & $\begin{array}{l}-1,133474 \\
(0,559)\end{array}$ & $\begin{array}{l}-1,342413 \\
(0,282)\end{array}$ & $\begin{array}{l}-0,9445218 \\
(0,551)\end{array}$ \\
\hline
\end{tabular}


TABLA 4 (cont.)

ESTIMACIONES PARA LA MUESTRA COMPLETA

\begin{tabular}{|l|l|l|l|}
\hline Q-ToBIN & \multicolumn{1}{|c|}{$(1)$} & \multicolumn{1}{|c|}{$(2)$} & \multicolumn{1}{|c|}{$(1-2)$} \\
\hline \multirow{2}{*}{ año 2006 } & $-1,081211$ & $-1,334158$ & $-0,7471503$ \\
& $(0,560)$ & $(0,271)$ & $(0,636)$ \\
\hline \multirow{2}{*}{ año 2005 } & $-1,248231$ & $-1,490439$ & $-0,8975478$ \\
& $(0,502)$ & $(0,222)$ & $(0,572)$ \\
\hline \multirow{2}{*}{ año 2004 } & $-1,387457$ & $-1,707425$ & $-1,189177$ \\
& $(0,456)$ & $(0,162)$ & $(0,454)$ \\
\hline \multirow{2}{*}{ año 2003 } & $-1,412772$ & $-1,602035$ & $-1,095124$ \\
& $(0,446)$ & $(0,195)$ & $(0,501)$ \\
\hline \multirow{2}{*}{ año 2002 } & $-1,266506$ & $-1,276016$ & $-1,005332$ \\
& $(0,242)$ & $(0,147)$ & $(0,309)$ \\
\hline Dimensión consejo & & & 0,4174 \\
óptima. & & 0,3179 & 0,5296 \\
Independencia consejo & & 0,4128 & \\
óptima. & & & $25,35(0,000)^{* * *}$ \\
Propiedad interna óptima & & $3,36(0,000)^{* * *}$ & $10,82(0,372)$ \\
\hline F- test & $36,42(0,000)^{\star * *}$ & $-0,06(0,512)$ & $-1,03(0,302)$ \\
Hansen test $\chi^{2}$ & $10,65(0,300)$ & $0,96(0,322)$ & $0,97(0,332)$ \\
AR(1) & $-0,98(0,328)$ & $-1,12(0,263)$ & \\
AR(2) & &
\end{tabular}

Estimaciones MGM de sistema en dos etapas con la corrección robusta para muestras finitas propuesta por Windmeijer (2005). Coeficientes estimados y $p>[t]$ de significatividad de los coeficientes entre paréntesis [1\% $\left(^{\star \star *}\right), 5 \%\left({ }^{* \star}\right)$ y $\left.10 \%\left({ }^{\star}\right)\right]$. La variable dependiente es la creación de valor para de la empresa aproximada a través de la Q-Tobin. Las variables explicativas son la dimensión del consejo (número de consejeros), su independencia (proporción de consejeros independientes sobre el total de consejeros) y la propiedad interna (proporción de derechos de propiedad de los consejeros). Las variables de control son el tamaño de la empresa (logaritmo del activo total), el nivel de endeudamiento (proporción de la deuda sobre el activo total) y las dummies temporales. El estadístico de Hansen se distribuye siguiendo una función $\chi^{2}$ con tantos grados de libertad como el número de coeficientes estimados.

Por otro lado, los coeficientes significativos de las variables independencia consejo e independencia consejo $^{2}$, positivo y negativo respectivamente, muestran una relación no lineal en forma de $U$ invertida entre la independencia del consejo y la variable dependiente, confirmando nuestra tercera hipótesis. Este resultado establece la existencia de un punto óptimo a partir del cual la incorporación de un nuevo consejero externo disminuye la creación de valor, lo que apoya el argumento de que una adecuada combinación de consejeros internos-consejeros externos es mejor que una excesiva independencia del consejo. Así, se pone de manifiesto la relevancia de la función de asesoramiento, que requiere la presencia de consejeros internos ejecutivos. Para la totalidad de la muestra, la proporción óptima de independientes es de un $41,74 \%$.

Respecto a la quinta hipótesis, la significatividad de la variable propiedad interna refleja el efecto positivo de la propiedad de los consejeros sobre la creación de valor, configurándose dicha propiedad como mecanismo sustitutivo de la supervisión ejercida por el consejo, lo que permite que éste se centre en su función de asesoramiento. Sin embargo, el signo negativo y significativo de la variable propiedad interna ${ }^{2}$ pone de relieve la existencia de un nivel óptimo de propiedad, que se sitúa, para el conjunto de la muestra en un $52,96 \%$, a partir del cual los problemas de atrincheramiento tienen una incidencia negativa sobre la creación de valor. Por tanto, para la totalidad de la muestra no podemos confirmar nuestra 
quinta hipótesis, que establecía una relación positiva entre la propiedad en manos de los consejeros y la variable dependiente.

\subsection{Resultados Del MOdelo de EFECTOS INTERACTIVOS}

En la Tabla 5 se presentan los modelos interactivos, donde los coeficientes de las variables interactivas recogen el efecto incremental que tienen cada una de las tres características del consejo consideradas ${ }^{(12)}$, dimensión, independencia y propiedad interna, sobre la creación de valor de las empresas familiares con respecto al grupo de referencia, las no familiares. $\mathrm{Su}$ significatividad pone de manifiesto la diferente influencia de estas tres características sobre la variable dependiente en función de la naturaleza familiar o no de la empresa. El coeficiente para las empresas familiares $(\Sigma)$ es la suma del coeficiente para las no familiares más el efecto interactivo. En este apartado no nos detenemos en la significatividad de las variables para la submuestra de empresas familiares, que es analizada en el tercer apartado, sino únicamente en la existencia de diferencias significativas con respecto a la no familiar.

En primer lugar, la falta de significatividad de las variables dimensión consejo y dimensión consejo ${ }^{2}$ no interactivas no nos permite asegurar la relación cuadrática con la creación de valor (hipótesis uno) para las no familiares, mientras que el coeficiente negativo y significativo del efecto incremental dimensión consejo fam cambia el signo positivo del coeficiente de la variable dimensión consejo y lo vuelve negativo para las empresas familiares $\left(\sum\right)$, lo que sugiere la existencia de problemas de coordinación y control planteados en nuestra segunda hipótesis.

Por otro lado, como muestran los coeficientes de las variables independencia consejo e independencia consejo ${ }^{2}$, positivo y negativo respectivamente, se mantiene para las empresas no familiares la relación en forma de U invertida entre la independencia del consejo y la variable dependiente encontrada para la muestra global, como establecía la hipótesis tercera. Sin embargo, el signo negativo y la significatividad de las variables interactivas independencia consejo fam e independencia consejo ${ }^{2}$ fam apuntan la existencia de una relación negativa para la empresa familiar, lo que sugiere la importante labor de asesoramiento ejercida por los consejeros internos en este tipo de estructuras empresariales, tal y como planteamos en nuestra hipótesis cuarta.

\section{TABLA 5}

ESTIMACIONES PARA LA MUESTRA COMPLETA CON EFECTOS INTERACTIVOS

\begin{tabular}{|l|r|l|l|l|l|l|}
\hline Q-Tobin & \multicolumn{2}{|c|}{$(3)$} & \multicolumn{2}{c|}{$(4)$} & \multicolumn{2}{c|}{$(3-4)$} \\
\hline constante & 2,234800 & $(0,137)$ & 1,946707 & $(0,244)$ & 2,736580 & $(0,147)$ \\
\hline dimensión consejo & 0,099340 & $(0,205)$ & 0,439816 & $(0,084)^{\star}$ & 0,125886 & $(0,439)$ \\
dimensión consejo fam & $-0,229916$ & $(0,034)^{\star \star}$ & $-0,639057$ & $(0,022)^{\star \star}$ & $-0,234736$ & $(0,069)^{\star}$ \\
$\Sigma$ & $-0,130575$ & $(0,100)^{*}$ & $-0,199240$ & $(0,059)^{*}$ & $-0,108849$ & $(0,073)^{*}$ \\
\hline
\end{tabular}

(Cont. pág. sig.)

(12) Debido a las diferencias de medias de las variables endeudamiento y tamaño empresa entre ambos grupos de compañías, se incorpora también el efecto interactivo para las mismas, para controlar las potenciales divergencias que puedan existir en su contribución a la creación de valor de la empresa. 
TABLA 5 (CONT.)

ESTIMACIONES PARA LA MUESTRA COMPLETA CON EFECTOS INTERACTIVOS

\begin{tabular}{|c|c|c|c|c|c|c|}
\hline Q-Tobin & \multicolumn{2}{|c|}{ (3) } & \multicolumn{2}{|c|}{ (4) } & \multicolumn{2}{|c|}{$(3-4)$} \\
\hline $\begin{array}{l}\text { dimensión consejo² } \\
\text { dimensión consejo fam } \\
\Sigma\end{array}$ & & & $\begin{array}{r}-0,011633 \\
0,016046 \\
0,004413\end{array}$ & $\begin{array}{l}(0,135) \\
(0,073)^{\star} \\
(0,348)\end{array}$ & & \\
\hline $\begin{array}{l}\text { independencia consejo } \\
\text { independencia consejo fam } \\
\Sigma\end{array}$ & $\begin{array}{r}1,022407 \\
-2,413471 \\
-1,391064\end{array}$ & $\begin{array}{l}(0,242) \\
(0,066)^{\star} \\
(0,179)\end{array}$ & $\begin{array}{r}5,456186 \\
-6,402837 \\
-0,946651\end{array}$ & $\begin{array}{l}(0,088)^{\star} \\
(0,061)^{\star} \\
(0,496)\end{array}$ & $\begin{array}{r}6,717573 \\
-8,780460 \\
-2,062887\end{array}$ & $\begin{array}{l}(0,021)^{\star \star} \\
(0,016)^{\star \star} \\
(0,043)^{\star \star}\end{array}$ \\
\hline $\begin{array}{l}\text { independencia consejo }{ }^{2} \\
\text { independencia consejo }{ }^{2} \text { fam } \\
\Sigma\end{array}$ & & & $\begin{array}{r}-6,653182 \\
6,124429 \\
-0,528753\end{array}$ & $\begin{array}{l}(0,063)^{\star} \\
(0,101) \\
(0,565)\end{array}$ & $\begin{array}{r}-8,762001 \\
8,287940 \\
-0,474061\end{array}$ & $\begin{array}{l}(0,016)^{\star *} \\
(0,061)^{\star} \\
(0,049)^{\star *}\end{array}$ \\
\hline $\begin{array}{l}\text { propiedad interna } \\
\text { propiedad interna fam } \\
\Sigma\end{array}$ & $\begin{array}{l}-0,107484 \\
-0,471957 \\
-0,579441\end{array}$ & $\begin{array}{l}(0,821) \\
(0,471) \\
(0,359)\end{array}$ & $\begin{array}{r}-1,173322 \\
4,692098 \\
3,518776\end{array}$ & $\begin{array}{l}(0,449) \\
(0,063)^{\star} \\
(0,076)^{\star}\end{array}$ & $\begin{array}{r}-0,999432 \\
4,631954 \\
3,632521\end{array}$ & $\begin{array}{l}(0,721) \\
(0,349) \\
(0,596)\end{array}$ \\
\hline $\begin{array}{l}\text { propiedad interna }{ }^{2} \\
\text { propiedad interna } 2 \text { fam } \\
\Sigma\end{array}$ & & & $\begin{array}{r}0,619811 \\
-4,728803 \\
-4,108992\end{array}$ & $\begin{array}{l}(0,690) \\
(0,069)^{\star} \\
(0,076)^{\star}\end{array}$ & $\begin{array}{r}0,785326 \\
-5,528327 \\
-4,743000\end{array}$ & $\begin{array}{l}(0,771) \\
(0,278) \\
(0,498)\end{array}$ \\
\hline $\begin{array}{l}\text { Endeudamiento } \\
\text { endeudamiento familiar } \\
\Sigma\end{array}$ & $\begin{array}{l}-1,076796 \\
-1,626250 \\
-2,703046\end{array}$ & $\begin{array}{l}(0,336) \\
(0,459) \\
(0,217)\end{array}$ & $\begin{array}{r}0,952670 \\
-3,781366 \\
-2,828695\end{array}$ & $\begin{array}{l}(0,399) \\
(0,064)^{\star} \\
(0,145)\end{array}$ & $\begin{array}{r}0,015033 \\
-2,784176 \\
-2,769142\end{array}$ & $\begin{array}{l}(0,987) \\
(0,151) \\
(0,245)\end{array}$ \\
\hline $\begin{array}{l}\text { tamaño empresa } \\
\text { tamaño empresa fam } \\
\Sigma\end{array}$ & $\begin{array}{l}0,000545 \\
0,336132 \\
0,336678\end{array}$ & $\begin{array}{l}(0,997) \\
(0,033)^{* *} \\
(0,076)^{*}\end{array}$ & $\begin{array}{r}-0,258648 \\
0,539304 \\
0,280655\end{array}$ & $\begin{array}{l}(0,258) \\
(0,010)^{* *} \\
(0,142)\end{array}$ & $\begin{array}{r}-0,130052 \\
0,383457 \\
0,253405\end{array}$ & $\begin{array}{l}(0,619) \\
(0,006)^{\star * *} \\
(0,017)^{\star *}\end{array}$ \\
\hline $\begin{array}{l}\text { año } 2007 \\
\text { año } 2006 \\
\text { año } 2005 \\
\text { año } 2004 \\
\text { año } 2003 \\
\text { año } 2002\end{array}$ & $\begin{array}{l}-1,663212 \\
-1,427569 \\
-1,658865 \\
-1,851382 \\
-1,802139 \\
-1,327511\end{array}$ & $\begin{array}{l}(0,054)^{\star} \\
(0,090)^{*} \\
(0,045)^{\star \star} \\
(0,027)^{\star *} \\
(0,030)^{\star *} \\
(0,032)^{\star *}\end{array}$ & $\begin{array}{l}-0,869892 \\
-0,875401 \\
-1,074270 \\
-1,354116 \\
-1,364512 \\
-1,073134\end{array}$ & $\begin{array}{l}(0,458) \\
(0,456) \\
(0,370) \\
(0,261) \\
(0,273) \\
(0,193)\end{array}$ & $\begin{array}{l}-1,329888 \\
-1,321560 \\
-1,524848 \\
-1,746744 \\
-1,709317 \\
-1,133688\end{array}$ & $\begin{array}{l}(0,299) \\
(0,283) \\
(0,220) \\
(0,156) \\
(0,160) \\
(0,204)\end{array}$ \\
\hline $\begin{array}{l}\text { F- test } \\
\text { Hansen test } X^{2} \\
\text { AR(1) } \\
\operatorname{AR}(2)\end{array}$ & $\begin{array}{r}2,98 \\
13,69 \\
-0,91 \\
0,66\end{array}$ & $\begin{array}{l}(0,001)^{\star * *} \\
(0,749) \\
(0,362) \\
(0,509)\end{array}$ & $\begin{array}{r}2,64 \\
54,60 \\
-0,94 \\
0,88\end{array}$ & $\begin{array}{l}(0,001)^{* * *} \\
(0,993) \\
(0,345) \\
(0,379)\end{array}$ & $\begin{array}{r}3,55 \\
41,59 \\
-0,91 \\
0,82\end{array}$ & $\begin{array}{l}(0,000)^{\star \star \star} \\
(0,401) \\
(0,365) \\
(0,411)\end{array}$ \\
\hline
\end{tabular}

Estimaciones MGM de sistema en dos etapas con la corrección robusta para muestras finitas propuesta por Windmeijer (2005). Coeficientes estimados y $p>[t]$ de significatividad de los coeficientes entre paréntesis $\left[1 \%\left({ }^{* * *}\right), 5 \%\left({ }^{* *}\right)\right.$ y $\left.10 \%\left({ }^{*}\right)\right]$. La variable dependiente es la creación de valor de la empresa aproximada a través de la Q-Tobin. Las variables explicativas son la dimensión del consejo (número de consejeros), su independencia (proporción de consejeros independientes sobre el total de consejeros) y la propiedad interna (proporción de derechos de propiedad de los consejeros). Las variables de control son el tamaño de la empresa (logaritmo del activo total), el nivel de endeudamiento (proporción de la deuda sobre el activo total) y las dummies temporales. Las variables interactivas (variables*fam) recogen el efecto incremental de cada una de las variables sobre la creación de valor de las empresas familiares con respecto al grupo de referencia, las no familiares. El coeficiente para las empresas familiares $\left(\sum\right)$ es la suma del coeficiente para las no familiares más el efecto interactivo, El estadístico de Hansen se distribuye siguiendo una función $\chi^{2}$ con tantos grados de libertad como el número de coeficientes estimados.

El efecto de la propiedad de los consejeros sobre la variable dependiente no resulta significativo para el grupo de referencia, la empresa no familiar, como puede verse en el coeficiente de la variable propiedad interna, que contrariamente a lo argumentado en nuestra quinta hipótesis es negativo. Sin embargo, los efectos incrementales de propiedad interna fam, positivo, y propiedad interna $a^{2}$ fam, negativo, que sí resultan estadísticamente significativos, sugieren la existencia de la relación no lineal planteada por nuestra sexta hipótesis entre la propiedad de los consejeros y la creación de valor de las empresas familiares. 


\subsection{Resultados Para las SUbMUESTRAS EMPRESAS Familiares y No FamiliareS}

Para corroborar los resultados obtenidos y analizar la significatividad de las variables en ambos grupos de empresas, realizamos un último análisis individual para cada submuestra. Así, para el grupo de empresas con estructura de propiedad concentrada no familiar contrastamos las hipótesis generales $H 1, H 3$ y $H 5$ [véanse columnas (5) y (6) de la Tabla 6], mientras que para las organizaciones de naturaleza familiar contrastamos las Hipótesis H2, H4 y H6 [véanse columnas (7) y (8) de la Tabla 6].

Respecto al efecto del tamaño del consejo sobre la variable dependiente, encontramos una relación no lineal en la submuestra de empresas no familiares que confirma nuestra primera hipótesis. Aunque la incorporación de nuevos consejeros está positivamente relacionada con la creación de valor de la empresa, como arroja el coeficiente positivo y significativo de la variable dimensión consejo, el incremento en la variable dependiente muestra un crecimiento marginal decreciente. Así, el signo negativo e igualmente significativo del coeficiente de la variable dimensión consejo ${ }^{2}$ pone de manifiesto la existencia de un nivel óptimo a partir del cual la incorporación de un nuevo consejero reduce la creación de valor. El valor que maximiza la función objetivo se sitúa en torno a 16 miembros. Por otra parte, la relación se confirma negativa para la empresa familiar (véase el coeficiente negativo y significativo de la variable dimensión consejo), en la que existen menores necesidades de supervisión por parte del consejo como consecuencia del efecto sustitutivo que ejerce la propiedad familiar, y donde los potenciales beneficios de un mayor tamaño derivados de la mayor capacidad asesora se ven superados por los problemas de coordinación, control y toma de decisiones asociados a los consejos sobredimensionados, tal y como plantea nuestra segunda hipótesis.

En cuanto a la independencia del consejo, el coeficiente positivo y significativo de la variable independencia consejo muestra un efecto positivo sobre la creación de valor de las empresas no familiares. Sin embargo, la significatividad del coeficiente negativo de la variable independencia consejo $^{2}$, pone de manifiesto la existencia de un límite a partir del cual el efecto se vuelve negativo, lo que nos permite aceptar la tercera hipótesis. Dicho límite se sitúa alrededor de un $42 \%$. Por otro lado, el coeficiente negativo y significativo de la variable independencia consejo encontrada para las empresas familiares pone de relieve la menor efectividad aportada por los consejeros externos en términos de supervisión y asesoramiento en esta clase de organizaciones, confirmándose nuestra cuarta hipótesis.

Finalmente, la relación entre la creación de valor de la empresa y la propiedad de los consejeros es negativa y no significativa para las compañías no familiares, como muestra el coeficiente de la variable propiedad interna, por lo que no podemos aceptar nuestra quinta hipótesis de alineamiento de intereses. Los coeficientes significativos de las variables propiedad interna y propiedad interna ${ }^{2}$, positivo y negativo respectivamente, muestran una relación en forma de U invertida para las empresas familiares que reafirma la hipótesis sexta, verificándose la existencia de un límite a la propiedad de los consejeros a partir del cual el efecto negativo del atrincheramiento familiar supera las ventajas de la alineación de intereses. Este nivel óptimo es de un 44\%, valor ligeramente superior al reportado por Anderson y Reeb (2003), que sitúan el punto de inflexión en torno a un tercio de la propiedad en manos de grupos familiares. 
Por último, respecto a las variables de control, el tamaño de la empresa mantiene una relación negativa con la creación de valor en las empresas no familiares y positiva en las familiares, que tienen una dimensión media significativamente menor. El nivel de endeudamiento resulta negativo en las familiares, mientras que en las no familiares el efecto resulta positivo aunque no significativo. Las dummies temporales, que no son significativas en los modelos generales, sí lo son generalmente en los modelos específicos para ambos subgrupos.

TABLA 6

ESTIMACIONES POR SUBMUESTRAS

\begin{tabular}{|c|c|c|c|c|}
\hline Q-Tobin & $E N F(5)$ & $E N F(6)$ & $E F(7)$ & $E F(8)$ \\
\hline constante & $\begin{array}{r}0,4983641 \\
(0,772)\end{array}$ & $\begin{array}{r}0,5441415 \\
(0,759)\end{array}$ & $\begin{array}{r}-0,6683078 \\
(0,664)\end{array}$ & $\begin{array}{r}-1,684208 \\
(0,310)\end{array}$ \\
\hline dimensión consejo & $\begin{array}{r}0,5036793 \\
(0,017)^{\star *}\end{array}$ & $\begin{array}{l}0.4505701 \\
(0.007)^{\star \star *}\end{array}$ & $\begin{array}{r}-0.1873907 \\
(0.022)^{\star *}\end{array}$ & $\begin{array}{r}-0.1518549 \\
(0.077)^{*}\end{array}$ \\
\hline dimensión consejo ${ }^{2}$ & $\begin{array}{r}-0,0154737 \\
(0,022)^{\star *}\end{array}$ & $\begin{array}{r}-0,0138169 \\
(0,028)^{\star *}\end{array}$ & & \\
\hline independencia consejo & $\begin{array}{l}3,814227 \\
(0,019)^{\star *}\end{array}$ & $\begin{array}{l}3,919029 \\
(0,050)^{\star *}\end{array}$ & $\begin{array}{l}-2,267686 \\
(0,010)^{\star *}\end{array}$ & $\begin{array}{r}-2,278469 \\
(0,029)^{\star *}\end{array}$ \\
\hline independencia consejo ${ }^{2}$ & $\begin{array}{r}-4,289429 \\
(0,078)^{*}\end{array}$ & $\begin{array}{r}-4,671731 \\
(0,059)^{*}\end{array}$ & & \\
\hline propiedad interna & $\begin{array}{r}0,2095098 \\
(0,927)\end{array}$ & $\begin{array}{r}-0,437860 \\
(0,407)\end{array}$ & $\begin{array}{r}-0,9256985 \\
(0,098)^{*}\end{array}$ & $\begin{array}{r}4,190864 \\
(0,095)^{*}\end{array}$ \\
\hline propiedad interna $^{2}$ & $\begin{array}{r}-0,9298124 \\
(0,701)\end{array}$ & & & $\begin{array}{r}-4,711375 \\
(0,068)^{*}\end{array}$ \\
\hline endeudamiento & $\begin{array}{r}0,7279116 \\
(0,532)\end{array}$ & $\begin{array}{r}0,6468359 \\
(0,461)\end{array}$ & $\begin{array}{c}-3,71095 \\
(0,012)^{\star *}\end{array}$ & $\begin{array}{c}-3,623513 \\
(0,011)^{\star *}\end{array}$ \\
\hline tamaño empresa & $\begin{array}{r}-0,294181 \\
(0,135)\end{array}$ & $\begin{array}{r}-0,2677858 \\
(1,118)\end{array}$ & $\begin{array}{l}0,6529553 \\
(0,002)^{* * *}\end{array}$ & $\begin{array}{l}0,6257971 \\
(0,002)^{\star \star *}\end{array}$ \\
\hline $\begin{array}{l}\text { año } 2007 \\
\text { año } 2006 \\
\text { año } 2005 \\
\text { año } 2004 \\
\text { año } 2003 \\
\text { año } 2002\end{array}$ & $\begin{array}{r}1,085276 \\
(0,001)^{\star * *} \\
0,9190845 \\
(0,000)^{\star \star \star} \\
0,7446998 \\
(0,001)^{\star \star \star} \\
0,6041826 \\
(0,022)^{\star *} \\
0,4859295 \\
(0,065)^{\star} \\
- \\
-\end{array}$ & $\begin{array}{r}1,031415 \\
(0,001)^{\star * *} \\
0,8942806 \\
(0,000)^{\star * *} \\
0,7502303 \\
(0,000)^{\star * *} \\
0,655116 \\
(0,003)^{\star \star *} \\
0,5474596 \\
(0,012)^{\star *} \\
- \\
-\end{array}$ & $\begin{array}{r}-1,670836 \\
(0,014)^{\star *} \\
-1,119521 \\
(0,090)^{\star} \\
-1,435712 \\
(0,038)^{\star *} \\
-1,661024 \\
(0,007)^{\star * *} \\
-1,530451 \\
(0,013)^{\star *} \\
-0,9084905 \\
(0,008)^{\star * *}\end{array}$ & $\begin{array}{r}-1,419482 \\
(0,171) \\
-1,109343 \\
(0,260) \\
-1,312602 \\
(0,205) \\
-1,611601 \\
(0,113) \\
-1,458533 \\
(0,150) \\
-0,8128265 \\
(0,262)\end{array}$ \\
\hline $\begin{array}{l}\text { Dimensión consejo optima } \\
\text { Independencia consejo } \\
\text { óptima } \\
\text { Propiedad interna óptima }\end{array}$ & $\begin{array}{r}16,28 \\
0,4446\end{array}$ & $\begin{array}{r}16,31 \\
0,4194\end{array}$ & & 0,4448 \\
\hline
\end{tabular}


TABLA 6 (CONT.)

ESTIMACIONES POR SUBMUESTRAS

\begin{tabular}{|c|c|c|c|c|}
\hline Q-Tobin & $E N F(5)$ & $\operatorname{ENF}(6)$ & $E F(7)$ & $E F(8)$ \\
\hline F- test & $12,62(0,000)^{* * *}$ & $15,21(0,000)^{* * *}$ & $6,61(0,000)^{* * *}$ & $5,58(0,000)^{* * *}$ \\
\hline Hansen test $\chi^{2}$ & $22,98(0,816)$ & $16,96(0,883)$ & $10,46(0,314)$ & $20,69(0,191)$ \\
\hline $\operatorname{AR}(1)$ & $0,38(0,707)$ & $0,39(0,699)$ & $-0,98(0,326)$ & $-1,01(0,314)$ \\
\hline $\operatorname{AR}(2)$ & $-0,28(0,780)$ & $-0,33(0,744)$ & $1,01(0,313)$ & $0,93(0,352)$ \\
\hline
\end{tabular}

Estimaciones MGM de sistema en dos etapas con la corrección robusta para muestras finitas propuesta por Windmeijer (2005). Coeficientes estimados y $p>[t]$ de significatividad de los coeficientes entre paréntesis $\left(1 \%\left(^{* * *}\right), 5 \%\left({ }^{* *}\right)\right.$ y $\left.10 \%\left({ }^{*}\right)\right)$. EF: empresas familiares. ENF: empresas no familiares. La variable dependiente es la creación de valor de la empresa aproximada a través de la $Q$-Tobin. Las variables explicativas son la dimensión del consejo (número de consejeros), su independencia (proporción de consejeros independientes sobre el total de consejeros) y la propiedad interna (proporción de derechos de propiedad de los consejeros). Las variables de control son el tamaño de la empresa (logaritmo del activo total), el nivel de endeudamiento (proporción de la deuda sobre el activo total) y las dummies temporales. El estadístico de Hansen se distribuye siguiendo una función $\chi^{2} \operatorname{con}$ tantos grados de libertad como el número de coeficientes estimados.

\subsection{Consistencia de los resultados}

Con el fin de comprobar la consistencia de nuestros resultados ante cambios en la variable dependiente, realizamos un último análisis en el que estimamos los tres modelos finales (1-2 para la muestra completa y 6 y 8 para las empresas no familiares y familiares respectivamente) utilizando como medida alternativa a la $Q$-Tobin la ratio valor de mercado de las acciones sobre su valor contable. En la Tabla 7 puede verse que los resultados son robustos ante cambios en la variable dependiente.

TABLA 7

ESTIMACIÓN ALTERNATIVA DE LOS MODELOS FINALES

\begin{tabular}{|c|c|c|c|}
\hline ratio valor & COMPLETA (8) & $E N F(9)$ & $E F(10)$ \\
\hline constante & $\begin{array}{l}-4,17723 \\
(0,522)\end{array}$ & $\begin{array}{l}-4,1100720 \\
(0,710)\end{array}$ & $\begin{array}{l}-3,518141 \\
(0,410)\end{array}$ \\
\hline dimensión consejo & $\begin{array}{l}-0,6184112 \\
(0,013)^{\star \star}\end{array}$ & $\begin{array}{l}3,256565 \\
(0,074)^{\star}\end{array}$ & $\begin{array}{l}-0,3337619 \\
(0,008)^{\star * \star}\end{array}$ \\
\hline dimensión consejo ${ }^{2}$ & & $\begin{array}{l}-0,103168 \\
(0,100)^{\star}\end{array}$ & \\
\hline independencia consejo & $\begin{array}{l}11,28482 \\
(0,076)^{*}\end{array}$ & $\begin{array}{l}3,919029 \\
(0,050)^{\star \star *}\end{array}$ & $\begin{array}{l}-3,000179 \\
(0,006)^{\star * *}\end{array}$ \\
\hline independencia consejo ${ }^{2}$ & $\begin{array}{l}-14,11157 \\
(0,100)^{*}\end{array}$ & $\begin{array}{l}-4,671731 \\
(0,059)^{*}\end{array}$ & \\
\hline propiedad interna & $\begin{array}{l}6,341319 \\
(0,100)^{\star}\end{array}$ & $\begin{array}{l}-0,437860 \\
(0,407)\end{array}$ & $\begin{array}{l}4,57551 \\
(0,100)^{\star}\end{array}$ \\
\hline propiedad interna ${ }^{2}$ & $\begin{array}{l}-6,113796 \\
(0,100)^{\star}\end{array}$ & & $\begin{array}{l}-4,996968 \\
(0,092)^{\star}\end{array}$ \\
\hline endeudamiento & $\begin{array}{l}-0,0635683 \\
(0,229)\end{array}$ & $\begin{array}{l}0,6468359 \\
(0,461)\end{array}$ & $\begin{array}{l}-2,398069 \\
(0,471)\end{array}$ \\
\hline tamaño empresa & $\begin{array}{l}0,8826556 \\
(0,055)^{*}\end{array}$ & $\begin{array}{l}-0,2677858 \\
(1,118)\end{array}$ & $\begin{array}{l}0,9747643 \\
(0,028)^{\star *}\end{array}$ \\
\hline
\end{tabular}


TABLA 7 (CONT.)

ESTIMACIÓN ALTERNATIVA DE LOS MODELOS FINALES

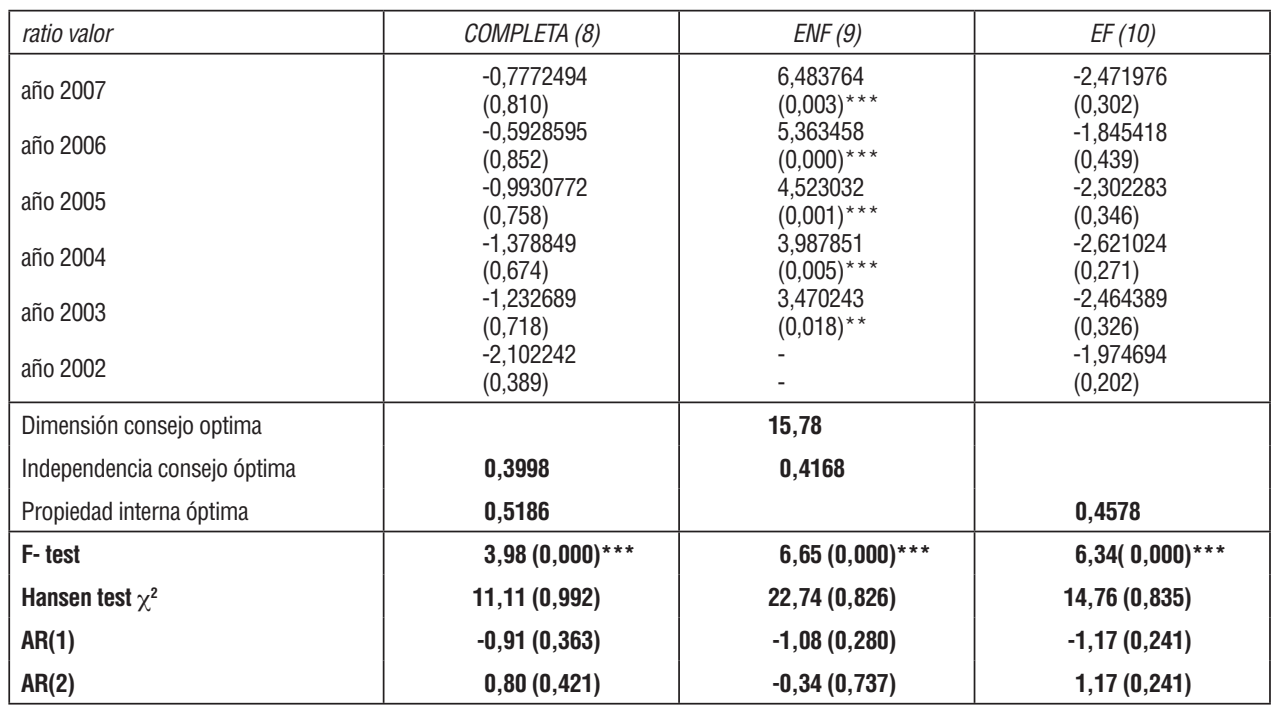

Estimaciones MGM de sistema en dos etapas con la corrección robusta para muestras finitas propuesta por Windmeijer (2005). Coeficientes estimados y $\mathrm{p}>$ [t] de significatividad de los coeficientes entre paréntesis $\left(1 \%\left(^{* * *}\right), 5 \%\left({ }^{* *}\right)\right.$ y $\left.10 \%\left({ }^{*}\right)\right)$. COMPLETA: muestra completa. EF: empresas familiares. ENF: empresas no familiares. La variable dependiente es la creación de valor de la empresa aproximada a través de la ratio valor de mercado de las acciones sobre su valor contable. Las variables explicativas son la dimensión del consejo (número de consejeros), su independencia (proporción de consejeros independientes sobre el total de consejeros) y la propiedad interna (proporción de derechos de propiedad de los consejeros). Las variables de control son el tamaño de la empresa (logaritmo del activo total), el nivel de endeudamiento (proporción de la deuda sobre el activo total) y las dummies temporales. El estadístico de Hansen se distribuye siguiendo una función $\chi^{2}$ con tantos grados de libertad como el número de coeficientes estimados.

En el caso de la muestra completa, el tamaño del consejo tiene un efecto negativo sobre la creación de valor, como indica el signo del coeficiente de la variable dimensión consejo. En cambio, tanto el nivel de independencia como el de propiedad interna tienen un efecto positivo sobre la creación de valor (véanse los signos de los coeficientes de las variables independencia consejo y propiedad interna), aunque los signos negativos de los coeficientes de las variables independencia consejo ${ }^{2}$ y propiedad interna ${ }^{2}$ muestran la existencia de un límite en dicho impacto positivo, que se sitúa en torno a un 40\% para el nivel de independencia y un $52 \%$ para la propiedad en manos de los consejeros.

Respecto a la submuestra de compañías no familiares, el signo de los coeficientes de las variables dimensión consejo e independencia consejo (positivos) y dimensión consejo ${ }^{2}$ e independencia consejo ${ }^{2}$ (negativos) confirman el límite al efecto positivo del número de consejeros y del nivel de independencia sobre la creación de valor de la empresa, resultando un tamaño óptimo próximo a los 16 miembros y una proporción óptima de consejeros independientes cercana al 42\%. Así mismo, no encontramos ninguna relación significativa de la propiedad interna con la variable dependiente.

Finalmente, en el grupo de empresas familiares corroboramos el efecto negativo que tanto la incorporación de un miembro adicional en el consejo (dimensión consejo) como el nivel de independencia (independencia consejo) tienen sobre la variable dependiente. Además, los signos de los coeficientes de las variables propiedad interna y propiedad interna ${ }^{2}$, po- 
sitivo y negativo respectivamente, ratifican el límite al impacto positivo que la propiedad en manos de los consejeros tiene sobre la creación de valor, con un nivel óptimo próximo al $46 \%$.

Adicionalmente, los modelos finales obtenidos, tanto para el caso de la $Q$ de Tobin (modelo 1-2 para la muestra completa y modelos 6 y 8 para las empresas no familiares y familiares respectivamente) como para el de la ratio valor (modelo 8 para la muestra completa y modelos 9 y 10 para las empresas no familiares y familiares respectivamente ), han sido estimados de forma alternativa sustituyendo las dummies temporales por las sectoriales Si bien éstas no resultaron estadísticamente significativas, los resultados obtenidos son similares a los reportados para las dummies temporales.

\section{CONCLUSIONES}

A lo largo del trabajo hemos realizado un análisis para una muestra de 85 empresas españolas no financieras en el período 2001-2007, que hemos dividido en dos subgrupos, familiares y no familiares. Teniendo en cuenta que el objetivo central del trabajo es atribuir las posibles diferencias en la eficiencia de los consejos de administración a la identidad familiar o no del accionista de control, hemos seleccionado una muestra lo más homogénea posible: todas las compañías cotizan en bolsa, tienen último propietario identificado y éste posee al menos un $25 \%$ de la propiedad. Nuestro punto de partida ha sido la identificación del último propietario de las acciones de la empresa a través del último eslabón de la cadena. Para la definición de la empresa familiar hemos utilizado no sólo la dimensión de la propiedad, sino también la del control, encontrando que un $40 \%$ de los casos analizados son empresas familiares, lo que confirma su importancia cuantitativa en España.

En segundo lugar hemos realizado un análisis integrado de dos de las funciones del consejo de administración, supervisión y asesoramiento, centrándonos en la incidencia que la estructura del mismo tiene sobre la creación de valor de la empresa. Mediante la metodología de datos de panel utilizada, hemos eliminado el problema de heterogeneidad inobservable y la endogeneidad de las variables explicativas consideradas, cuya omisión ha sido frecuente en los trabajos empíricos previos, conduciendo a estimaciones inconsistentes y difíciles de interpretar.

Este estudio nos ha permitido contrastar las distintas necesidades de supervisión y asesoramiento de las empresas según la identidad familiar del accionista de control mayoritario, lo que conduce a diferentes estructuras óptimas de gobierno. Del análisis se deduce que, de cara a la maximización de la creación de valor, las empresas han de elegir para sus consejos la estructura que mejor se adapte a su entorno contractual particular, sin que pueda afirmarse la existencia de una configuración óptima que se adapte a todas ellas. Así, la investigación llevada a cabo puede tener importantes implicaciones para la elaboración de las recomendaciones de buen gobierno, que creemos no deben ser homogéneas, sino que han de adaptarse a las características heterogéneas de las corporaciones cotizadas. En este sentido, nuestros resultados contradicen la creencia generalizada de que los consejos de administración pequeños, independientes y con una moderada participación de los consejeros en la propiedad de la empresa son siempre más eficientes. Como señala 
Cuervo-Cazurra (1998), las reformas de este órgano de gobierno que se están debatiendo en España podrían estar muy influenciadas por las desarrolladas en Estados Unidos y el Reino Unido, sin tener en cuenta que la existencia de problemas de separación de propiedad y control no parece justificarse en el contexto español salvo para un número de empresas muy reducido.

A pesar de que la incorporación de nuevos consejeros mejora las dos funciones del consejo consideradas, supervisión y asesoramiento, traduciéndose este efecto en la creación de valor de la empresa, existe un tamaño óptimo a partir del cual los problemas de coordinación, control y flexibilidad en la toma de decisiones asociados a los consejos sobredimensionados superan estos beneficios. Podemos concluir, en primer lugar, que el tamaño óptimo del consejo de administración viene definido por el trade-off existente entre las ventajas (mejor supervisión y asesoramiento) y las desventajas (problemas de coordinación y control) y, en segundo término, de acuerdo con Adams y Mehran (2005) y Andrés y Vallelado (2008), que las argumentaciones a favor de consejos pequeños no son siempre adecuadas cuando se consideran otras funciones de los consejos de administración, además de la tradicional labor supervisora.

La relación negativa encontrada entre el tamaño del consejo y la creación de valor en las empresas familiares puede sugerir que éstas han sobrepasado la dimensión óptima, impidiendo que la relación positiva existente antes de alcanzar el óptimo sea empíricamente observada. Uno de los motivos que podría justificar la persistencia de consejos sobredimensionados en este tipo de estructuras empresariales es el cumplimiento de la recomendación de independencia. Es decir, logran una «proporción adecuada» de consejeros independientes aumentando el tamaño de sus consejos en lugar de reestructurar los existentes, tradicionalmente compuestos en su mayoría por consejeros internos ejecutivos miembros de la unidad familiar. Por otro lado, el tamaño óptimo obtenido para los consejos de las empresas con estructura de propiedad concentrada no familiar se sitúa por encima del máximo establecido por estas recomendaciones.

Nuestro estudio también enfatiza el diferente papel desempeñado por la independencia del consejo en función de la naturaleza familiar o no del último propietario. A pesar de la evidencia empírica encontrada a favor de los consejos de administración independientes, tal y como habitualmente recomiendan los códigos de buen gobierno, hallamos que en las empresas con estructura de propiedad concentrada no familiar dicha independencia ha de tener un límite, por lo que una adecuada combinación de consejeros externos-consejeros internos contribuirá de forma más efectiva a la creación de valor que una excesiva independencia. Nuestros resultados refuerzan la hipótesis de que la información y el conocimiento que los consejeros internos aportan al consejo de administración son importantes para lograr una mayor eficiencia. Dicha importancia es especialmente relevante en las empresas familiares, en las que el conocimiento y la experiencia familiar aportados por estos consejeros contribuyen al desempeño eficaz de la función de asesoramiento. No obstante, aunque esperábamos encontrar un nivel óptimo de independencia inferior en las empresas familiares, según las argumentaciones teóricas expuestas en el segundo apartado de este trabajo consideramos que la participación de ejecutivos también ha de tener un límite para el eficaz desempeño de la función de supervisión, por lo que el hecho de haber 
encontrado una incidencia negativa de la independencia del consejo sobre la creación de valor nos hace cuestionarnos si en este tipo de organizaciones los consejeros independientes cumplen realmente con dicha condición de independencia, o si por el contrario el efecto negativo se debe a que éstas han adoptado niveles de independencia por encima del óptimo.

La participación del consejo en la propiedad de la empresa también tiene un impacto diferente en función de la identidad del accionista de control mayoritario. En las familiares la propiedad de los internos se confirma como un mecanismo sustitutivo de la supervisión ejercida por el consejo, lo que permite que éste se centre en su función asesora, con el consiguiente aumento de la eficiencia. Dicha propiedad ha de tener un límite, a partir del cual la posibilidad de un atrincheramiento familiar supera el efecto positivo comentado. Para las empresas no familiares, en las que la participación de los consejeros en la propiedad es menor, no se ha encontrado ninguna relación significativa.

En última instancia, es la adopción de aquella estructura del consejo de administración que mejor se adapte a las necesidades específicas de supervisión y asesoramiento de la organización la que determina la mayor o menor eficiencia del consejo y, por tanto, la mayor creación de valor de la empresa. Nuestra principal conclusión es que en las empresas familiares la labor de asesoramiento del consejo predomina sobre la de supervisión, cobrando una especial relevancia el papel de los consejeros internos.

El análisis llevado a cabo apunta la necesidad de seguir profundizando desde el ámbito académico en las diferencias existentes entre las empresas familiares y no familiares en sus prácticas y necesidades de gobierno. El correcto funcionamiento de los mecanismos de gobierno en general y del consejo de administración en particular puede contribuir a la supervivencia de este tipo de organizaciones empresariales. Como futuras ampliaciones de esta investigación se plantea la utilización de otras medidas de la estructura de los consejos, la construcción de una escala que recoja el diferente grado de implicación familiar en la empresa, así como la extensión del estudio a un entorno internacional.

\section{REFERENCIAS BIBLIOGRÁFICAS}

Acero, I., y Alcalde, N. 2008. Determinantes del tamaño y la estructura de los consejos de administración en las sociedades españolas cotizadas. XVIII Congreso Nacional de Acede, León, España. Septiembre.

Adams, R. B,, y Ferreira, D. 2007. A theory of friendly boards. The Journal of Finance LXII (1): 217-250.

Adams, R. B., y Mehran, H. 2005. Corporate performance board structure and its determinants in the banking industry. Working Paper, SSRN. Disponible en http://ssrn.com/abstract=302593 [acceso el 10 de enero de 2011].

Ampenberger, M.; Schmid, T.; KaSerer, C., y Achleitner, A. 2009. Capital structure and payout policy decisions in family businesses-empirical evidence from a bank-based economy. Working Paper Series, Center for Entrepreneurial and Financial Studies, Technische Universität München. Disponible en http://ssrn.com/abstract=1364153 [acceso el 10 de enero de 2011]. 
Anderson, R. C., y Reeb, D. M. 2003. Founding-family ownership and firm performance: evidence from the S\&P 500. The Journal of Finance LVIII (3): 1.301-1.328.

Anderson, R. C.; Mansi, S. A., y Reeb, D. M. 2003. Founding family ownership and the agency cost of debt. Journal of Financial Economics 68: 263-285.

Andrés, P., y Rodríguez, J. A. 2008. Friendly or controlling boards? XVIII Congreso Nacional de Acede, León, España. Septiembre.

Andrés, P., y VAlLELAdo, E. 2008. Corporate governance in banking: the role of the board of directors. Journal of Banking y Finance 32: 2.570-2.580.

Arellano, M., y Bond, S. 1991. Some tests of specification for panel data: Monte Carlo evidence and an application to employment equations. Review of Economic Studies 58: 277-297.

BARONTINI, R., y CAPRIO, L. 2005. The effect of family control on firm value and performance: Evidence from Continental Europe. Finance Working Paper, (88/2005). European Corporate Governance Institute.

Bartholomeusz, S., y TANewSKi, G. A, 2006. The relationship between family firms and corporate governance. Journal of Small Business Management 44 (2): 245-267.

BAYSINGER, B., y ButLER, H. 1985. Corporate governance and the board of directors: performance effects of changes in board composition. Journal of Law, Economics, and Organization 1: 101-124.

Berle, A. A., y MeAns, G. C. 1932. The modern corporation and private property. New York, Macmillan publishing co.

BLundell, R., y Bond, S.R. 1998. Initial conditions and moment restrictions in dynamic panel data models. Journal of Econometrics 87: 111-143.

Bond, S. R. 2002. Dynamic panel data models: A guide to micro data methods and practice. Working Paper 09/02, Institute for Fiscal Studies, London.

Boone, A.; Field, L.; KARPoff, J., y RAHEJA, C. 2007. The determinants of corporate board size and composition: An empirical analysis. Journal of Financial Economics 85: 66-101.

Business Roundtable, 1990 y 2002. Principles of corporate governance. Disponible en http:// www.businessroundtable.org [Acceso el 5 de mayo de 2010].

Caprio, G.; Laeven, L., y Levine, R. 2007. Governance and bank valuation. Journal of Financial Intermediation 16: 584-617.

CHENG, S. 2008. Board size and the variability of corporate performance. Journal of Financial Economics 87: 157-176.

Chung, K. H., y Pruitr, S. W. 1994. A simple approximation of Tobin's q. Financial Management 23: $70-74$

Claessens, S.; Duankov, S., y Lang, L. 2000. The separation of ownership and control in East Asian corporations. Journal of Financial Economics 58: 81-112.

Coles, J. L.; Daniel, N. D., y Naveen, L. 2008. Boards: Does one size fit all? Journal of Financial Economics 87 (2): 329-356.

Corbetta, G., y Salvato, C. 2004. The board of directors in family firms: one size fits all? Family Business Review XVII (2): 119-134.

Cuervo-Cazurra, A. 1998. La reforma del consejo de administración en España: Límites a la aplicación de los modelos anglosajones. Información Comercial Española 769: 9-22.

Daily, C. M., y Dalton, D. R. 1993. Board of directors leadership and structure control and performance implications. Entrepreneurship Theory and Practice Spring: 65-81. 
Demsetz, H. 1983. The structure of ownership and the theory of the firm. The Journal of Law and Economics 26: 75-390.

DemsetZ, H., y LeHN, K. 1985. The structure of corporate ownership: causes and consequences. The Journal of Political Economy 93 (6): 1.155-1.177.

Demsetz, H., y Villalonga, B. 2001. Ownership structure and corporate performance. Journal of Corporate Finance 7: 209-233.

Díaz, B., y García ,M. 2004. Asymmetric information and monitoring behaviour in block trades: an empirical analysis for Spain. Journal of Corporate Ownership \& Control 2 (1): 25-37.

DYER, W. G. 2003. The family: the missing variable in organizational research. Entrepreneurship Theory and Practice 27 (4): 401-416.

Eisenberg, T.; Sundgren, S., y Wells, M. T. 1998. Larger board size and decreasing firm value in small firms. Journal of Financial Economics 48: 35-54.

Faccio, M., y LANG, L. H. P. 2002. The ultimate ownership of Western European corporations. Journal of Financial Economics 65: 365-395.

FAma, E. F., y Jensen, M. C. 1983. Agency problems and residual claims. The Journal of Law and Economics 26 (2): 327-349.

FERNÁNDEZ, Z., y NiETo M. J. 2001. Internationalization strategy of small and medium-sized family businesses: Some influential factors. Family Business Review 18 (1): 77-88.

Fond, R. H. 1992. Boards of directors and the privately owned firm. New York: Quorum Books.

Galve, C., y SAlas, V. 2003. La empresa familiar en España. Fundamentos económicos y resultados. Ed. Fundación BBVA.

GARCíA, B., y GilL, B. 2005. El Gobierno Corporativo y las prácticas de earnings management: Evidencia empírica en España. Working Paper WP-EC-2005-11, Instituto Valenciano de Investigaciones Económicas.

GedaJlovic, E., y Shapiro, D. M. 1998. Management and ownership effects: evidence for five countries. Strategic Management Journal 19: 533-553.

Gómez-MeJía, L.; LARRAzA, M., y MaKRi, M. 2003. The determinants of executive compensation in family-controlled public corporations. Academy of Management Journal 46: 226-237.

Harris, M., y Raviv, A. 2008. A theory of board control and size. The Review of Financial Studies 21 (4): 1.797-1.833.

Hermalin, B. E., y Weisbach, M. S. 1991. The effect of board composition and direct incentives on firm performance. Financial Management 20: 101-112.

Hermalin, B. E., y Weisbach, M. S. 1998. The determinants of board composition. The Journal of Economics 19 (4): 589-606.

Hermalin, B. E., y Weisbach, M. S. 2003. Board of directors as an endogenously determined institution. Federal Reserve Bank of New York Economic Policy Review 9: 1-20.

HusE, M. 2005. Accountability and creating accountability: a framework for exploring behavioural perspectives of corporate governance. British Journal of Management 16: 65-79.

Jensen, M. C., y Meckling, W. H. 1976. Theory of the firm: managerial behavior, agency costs and ownership structure. Journal of Financial Economics 3 (4): 305-360.

KLEIN, A. 1998. Firm performance and board committee structure. Journal of Law and Economics 41 (1): 275-303.

La Porta, R.; Lopez-De-Silanes, F., y Shleifer, A. 1999. Corporate ownership around the world. The Journal of Finance 54 (2): 471-517. 
Levinson, H. 1971. Conflicts that plague family businesses. Harvard Business Review 49 (2): 90-98. Linck, J. S; NetTer, J. M., y YANG, T. 2008. The determinants of board structure. Journal of Financial Economics 87 (2): 308-328.

LODERER, C., y MARTIN, K. 1997. Executive stock ownership and market valuation: an empirical analysis. Journal of Financial Economics 45 (2): 223-255.

MAUG, E. 1996. Ownership structure as a determinant of IPO underpricing: a theory of the decision to go publicfor venture capital backed companies. Working paper, London Business School.

Maury, B., y Pajuste, A. 2005. Multiple large shareholders and firm value. Journal of Banking y Finance 29: 1.813-1.834.

McVey, H.; Draho, J., y Stanley, M. 2005. U.S. family-run companies- they may be better than you think. Journal of Applied Corporate Finance 17 (4): 134-144.

MíNGUEZ, A. 2005. Concentración accionarial y consejo de administración como mecanismos de control interno: Cuatro ensayos. Revista Española de Financiación y Contabilidad / Spanish Journal of Finance and Accounting, 125: 551-554.

Мок, H. M. K.; LAM, K., y CheUnG, I. 1992. Family control and return covariation in Hong Kong's common stocks. Journal of Business, Finance y Accounting, 19 (2): 277-293.

Mroczkowski, N. A., y TANewski, G. 2007. Delineating publicy listed family and non-family controlled firms: an approach for capita market research in Australia. Journal of Small Business Management 45 (3): 320-332.

PeArce, J. A., y Zahra, S. A. 1992. Board compensation from a strategic contingency perspective. Journal of Management Studies 29: 411-438.

Perfect, S. B., y WiLEs, K. W. 1994. Alternative constructions of Tobin's q: an empirical comparison. Journal of Empirical Finance 1: 313-341.

Pindado, J.; Requejo, I., y De la TorRe, C. 2008. Does family ownership impact positively on firm value? Empirical evidence from Western Europe. XVI Foro de Finanzas, Barcelona, España. Noviembre.

RAHEJA, C. 2005. Determinants of board size and composition: A theory of corporate boards. Journal of Financial and Quantitative Analysis 40: 283-206.

Roodman, D. 2009. A note on the theme of too many instruments. Oxford Bulletin of Economics and Statistics 71 (1): 135-158.

Rosenstein, S., y Wyatt, J. 1997. Inside directors, board effectiveness and shareholder wealth. Journal of Financial Economics 44: 229-250.

SACRISTÁN, M., y Gómez, S. 2007. Family ownership and pyramids in the Spanish market. Family Business Review 20 (3): 247-265.

Santana, D. J., y Aguiar, I. 2006. El último propietario de las empresas cotizadas españolas (1996-2002). Cuadernos de Economía y Dirección de la Empresa 26: 47-72.

SANTANA, D. J., y CABRERA, K. 2001. Comportamiento y resultados de las empresas cotizadas familiares versus no familiares. XI Congreso Nacional de Acede, Zaragoza, España. Septiembre.

Schulze, W. S.; Lubatkin, M. H. y Dino, R. N. 2003. Exploring the agency consequences of ownership dispersion among the directors of private family. The Academy of Management Journal 46 (2): 179-194.

ShleIfER, A., y Vishny, R. W. 1986. Large shareholders and corporate control. Journal of Political Economy 94: 461-488. 
Thomsen, S. y Pedersen, T. 2000. Ownership structure and economic performance in the largest European companies. Strategic Management Journal 21: 689-705.

TsaI, W. H.; HunG, J. H.; Kuo, Y. C., y Kuo, L. 2006. CEO tenure in Taiwanese family and non-family firms: an agency theory perspective. Family Business Review 19 (1): 11-28.

Villalonga, B., y Амiт, R. 2006. How do family ownership, control and management affect firm value? Journal of Financial Economics 80 (2): 385-417.

Voordeckers, W.; VAn Gils, A., y VAn den Heuvel, J. 2007. Board composition in small and medium-sized family firms. Journal of Small Business Management 45 (1): 137-15.

WindMEIJER, F. 2005. A finite sample correction for the variance of linear efficient two-step GMM estimators. Journal of Econometrics 126: 25-51.

YeRMACK, D. 1996. Higher market valuation of companies with a small board of directors. Journal of Financial Economics 40: 185-211. 